\title{
Construction of $\mathrm{SO}(5) \supset \mathrm{SO}(3)$ spherical harmonics and Clebsch-Gordan coefficients
}

\author{
M. A. Caprio, ${ }^{1}$ D. J. Rowe, ${ }^{2}$ and T. A. Welsh ${ }^{2}$ \\ ${ }^{1}$ Department of Physics, University of Notre Dame, Notre Dame, Indiana 46556-5670, USA \\ ${ }^{2}$ Department of Physics, University of Toronto, Toronto, Ontario M5S1A7, Canada
}

(Dated: February 3, 2009)

\begin{abstract}
The $\mathrm{SO}(5) \supset \mathrm{SO}(3)$ spherical harmonics form a natural basis for expansion of nuclear collective model angular wave functions. They underlie the recently-proposed algebraic method for diagonalization of the nuclear collective model Hamiltonian in an $\mathrm{SU}(1,1) \times \mathrm{SO}(5)$ basis. We present a computer code for explicit construction of the $\mathrm{SO}(5) \supset \mathrm{SO}(3)$ spherical harmonics and use them to compute the Clebsch-Gordan coefficients needed for collective model calculations in an $\mathrm{SO}(3)$-coupled basis. With these Clebsch-Gordan coefficients it becomes possible to compute the matrix elements of collective model observables by purely algebraic methods.
\end{abstract}

\section{Program Summary}

Title of program: GammaHarmonic

Catalogue identifier: AECY_v1_0

Program summary URL: http://cpc.cs.qub.ac.uk/summaries/AECY_v1_0

Program available from: CPC Program Library, Queen's University of Belfast, N. Ireland

Operating system: Any which supports Mathematica; tested under Microsoft Windows XP and Linux

Programming language used: Mathematica 6

Number of bytes in distributed program, including test code and documentation: 16037234

Distribution format: tar.gz

Nature of problem: Explicit construction of $\mathrm{SO}(5) \supset \mathrm{SO}(3)$ spherical harmonics on $S_{4}$. Evaluation of $\mathrm{SO}(3)$-reduced matrix elements and $\mathrm{SO}(5) \supset \mathrm{SO}(3)$ Clebsch-Gordan coefficients (isoscalar factors).

Method of solution: Construction of $\mathrm{SO}(5) \supset \mathrm{SO}(3)$ spherical harmonics by orthonormalization, obtained from a generating set of functions, according to the method of D. J. Rowe, P. S. Turner, and J. Repka [J. Math. Phys. 45 (2004) 2761]. Matrix elements and Clebsch-Gordan coefficients follow by construction and integration of SO(3) scalar products.

PACS: 02.20.Qs, 02.70.-c, 21.60.Ev, 21.60.Fw

Keywords: $\mathrm{SO}(5)$; spherical harmonics; Clebsch-Gordan coefficients; coupling coefficients; isoscalar factors; Bohr Hamiltonian; $\mathrm{SU}(1,1) \times \mathrm{SO}(5)$ algebraic collective model

\section{INTRODUCTION}

The $\mathrm{SO}(5) \supset \mathrm{SO}(3)$ spherical harmonics constitute the natural basis for the "angular" wave functions in the collective model of nuclear quadrupole motion [1]. The $\mathrm{SO}(5) \supset \mathrm{SO}(3)$ spherical harmonics underlie the recentlyproposed algebraic scheme [2-4] for the nuclear collective model. Direct products of the $\mathrm{SO}(5) \supset \mathrm{SO}(3)$ spherical harmonics with appropriate optimal radial wave functions provide an $\mathrm{SU}(1,1) \times \mathrm{SO}(5)$ algebraic basis $[2-4]$ which allows for exceedingly efficient numerical diagonalization of nuclear collective model Hamiltonians.

For applications to transitional and deformed nuclei, the $\mathrm{SU}(1,1) \times \mathrm{SO}(5)$ scheme reduces by orders of magnitude [3] the basis size needed for convergence as compared to conventional diagonalization in a five-dimensional oscillator $[\mathrm{U}(5) \supset \mathrm{SO}(5)]$ basis [5-7]. Matrix elements of an essentially unlimited set of potential and kinetic energy operators, often in analytic form, can easily be constructed in an $\mathrm{SU}(1,1) \times \mathrm{SO}(5)$ basis $[3,4]$, in terms of $\mathrm{SO}(5) \supset \mathrm{SO}(3)$ Clebsch-Gordan coefficients. The resulting calculational scheme, the so-called algebraic collective model (ACM), is described in Refs. [2-4, 8]. Examples of physical applications may be found in Ref. [9].

In this article, we present a computer code for explicit construction of the $\mathrm{SO}(5) \supset \mathrm{SO}(3)$ spherical harmonics and for using them to determine the Clebsch-Gordan coefficients for coupling of symmetric irreducible representations of $\mathrm{SO}(5)$ in an $\mathrm{SO}(3)$ basis. The construction of the $\mathrm{SO}(5) \supset \mathrm{SO}(3)$ spherical harmonics is carried out by 
orthonormalization of monomials in a set of four generating functions, according to the method of Rowe, Turner, and Repka [10]. The purpose of this code is to make calculations using the ACM routinely possible, without the need to reconstruct the $\mathrm{SO}(5)$ machinery for each new application. The $\mathrm{SO}(5) \supset \mathrm{SO}(3)$ Clebsch-Gordan coefficients yielded by the code are also relevant to the $\mathrm{U}(6)$ interacting boson model (IBM) $[11,12]$ which, in $\mathrm{U}(6) \supset \mathrm{U}(5) \supset \mathrm{SO}(5)$ and $\mathrm{U}(6) \supset \mathrm{SO}(6) \supset \mathrm{SO}(5)$ bases, is in close correspondence with the collective model in appropriate $\mathrm{SU}(1,1) \times \mathrm{SO}(5)$ bases [13]. They can, furthermore, be used as seed coefficients in the generation of Clebsch-Gordan coefficients for the coupling of more general (non-symmetric) representations of $\mathrm{SO}(5)$ or $\mathrm{Sp}(4)$ in an $\mathrm{SO}(3)$ basis [14].

The code accompanying this article is implemented in Mathematica 6 [15]. All calculations are carried out in exact symbolic arithmetic. A machine-readable tabulation of calculated Clebsch-Gordan coefficients is included along with the code in the CPC Program Library. This tabulation is of sufficient extent to support basic calculations using the algebraic collective model, without the necessity of re-running the code.

The basic algorithm used here is that presented in Ref. [10]. However, the computational techniques have been substantially developed. For example, integration via the Fourier representation of functions has been used to greatly increase the efficiency of the calculations and considerably extend the practical range of application of the algorithm.

The necessary mathematical definitions for the $\mathrm{SO}(5) \supset \mathrm{SO}(3)$ spherical harmonics and the general method for construction of the basis and evaluation of Clebsch-Gordan coefficients are discussed in Sec. 2. The more technical details of the computer implementation are summarized in Sec. 3. Instructions for installation and use of the computer code are given in Sec. 4 .

\section{THE BASIC ALGORITHM}

\subsection{The $\mathrm{SO}(5) \supset \mathrm{SO}(3)$ spherical harmonics}

The $\mathrm{SO}(5)$ spherical harmonics are eigenfunctions of the Laplace-Beltrami operator $\hat{\Lambda}^{2}$ on the four-sphere $S_{4}$, that is, the angular part of the Laplacian in five dimensions. This operator $\hat{\Lambda}^{2}$ is also the second order Casimir invariant of $\mathrm{SO}(5)$. Thus, the spherical harmonics are functions of a set of coordinates on $S_{4}$. Standard $(\gamma, \Omega)$ coordinates for $S_{4}$ are reviewed below in Sec. 2.2. The $\mathrm{SO}(5)$ spherical harmonics constitute a complete orthonormal basis for the space $L^{2}\left(S_{4}\right)$ of square-integrable functions on $S_{4}$ and transform under $\mathrm{SO}(5)$ rotations as bases for the symmetric irreps $(v, 0)$, for $v=0,1, \ldots$, of $\mathrm{SO}(5)$.

For applications to the nuclear collective model, we seek spherical harmonics which have "good $\mathrm{SO}(3)$ angular momentum", that is, which also transform as bases for irreps, labelled by $(L)$, of the $\mathrm{SO}(3)$ subalgebra of $\mathrm{SO}(5)$. The desired $\mathrm{SO}(5) \supset \mathrm{SO}(3)$ spherical harmonics, which we denote by $\Psi_{v \alpha L M}(\gamma, \Omega)$, thus reduce the subalgebra chain

$$
\underset{v}{\mathrm{SO}}(5) \underset{\alpha}{\supset} \underset{L}{\mathrm{SO}}(3) \underset{M}{\supset} \underset{\mathrm{SO}}{\mathrm{S}}(2),
$$

with the representation labels as shown. The label $v$ is conventionally termed the "boson seniority" quantum number, following Racah. It is the five-dimensional $[\mathrm{SO}(5)]$ analog of the angular momentum quantum number. The $\mathrm{SO}(5)$ spherical harmonics satisfy the eigenvalue equation

$$
\hat{\Lambda}^{2} \Psi_{v \alpha L M}(\gamma, \Omega)=v(v+3) \Psi_{v \alpha L M}(\gamma, \Omega) .
$$

They are also eigenfunctions of the Casimir operators of the other algebras in the chain (1). However, the labels $v L M$ provided by these Casimir operators are insufficient to fully distinguish the spherical harmonics. In particular, multiple $\mathrm{SO}(3)$ representations of the same $L$ may occur within a given $\mathrm{SO}(5)$ representation. The "missing label" is provided by a multiplicity index $\alpha$. The branching rule for $\mathrm{SO}(3)$ irreps occurring within an $\mathrm{SO}(5)$ irrep is well known $[16,17]$, and the multiplicity is given by $(\mathrm{A} 1)$.

The $\mathrm{SO}(5) \supset \mathrm{SO}(3)$ spherical harmonics have physical significance as the angular, i.e., $(\gamma, \Omega)$, wave functions for the nuclear collective model, for the case in which which the collective potential is $\mathrm{SO}(5)$ invariant $[18,19]$. Recall the quantum mechanics of a particle in three-dimensional Euclidean space, subject to a central force, i.e., in spherical coordinates, $V(r, \theta, \varphi) \rightarrow V(r)$. The Hamiltonian is then $\mathrm{SO}(3)$ invariant, and its eigenfunctions, with good angular momentum quantum numbers, factorize into products $f_{n l}(r) Y_{l m}(\theta, \varphi)$ of radial wave functions and $\mathrm{SO}(3)$ spherical harmonics. Similarly, for the Bohr Hamiltonian, which is given in terms of quadrupole deformation variables $\beta, \gamma$, and $\Omega$ (Sec. 2.2) by

$$
-\frac{\hbar^{2}}{2 B}\left[\frac{1}{\beta^{4}} \frac{\partial}{\partial \beta} \beta^{4} \frac{\partial}{\partial \beta}-\frac{\hat{\Lambda}^{2}}{\beta^{2}}\right]+V(\beta, \gamma),
$$

if the potential is a function $V(\beta)$ of the radial coordinate only, then the five-dimensional analog of a central force problem arises. The Hamiltonian is $\mathrm{SO}(5)$ invariant, and its eigenfunctions, with good seniority and angular momentum quantum numbers, factorize into products $f_{n v}(\beta) \Psi_{v \alpha L M}(\gamma, \Omega)$ of radial, i.e., $\beta$, wave functions and $\mathrm{SO}(5)$ spherical harmonics, as in Ref. [18]. An example of application of the present methods to such " $\gamma$-unstable" problems is found in Ref. [14].

A limited set of $\mathrm{SO}(5) \supset \mathrm{SO}(3)$ spherical harmonics was computed many years ago by Bès [19], for values of the angular momentum $L \leq 6$. These were obtained by series solution of coupled differential equations in the coordinate $\gamma$, to find the eigenfunctions of the $\mathrm{SO}(5)$ Casimir invariant. However, this approach becomes prohibitively 
complicated for $L>6$. The $\mathrm{SO}(5) \supset \mathrm{SO}(3)$ spherical harmonics are the angular wave functions for the five-dimensional quadrupole harmonic oscillator [20, 21]. Thus, alternative approaches based on the oscillator basis construction are possible, as summarized in Ref. [7]. A method based on the Cartan-Weyl reduction is given in Ref. [22].

Here we make use of the construction proposed in Ref. [10], in which the $\mathrm{SO}(5) \supset \mathrm{SO}(3)$ spherical harmonics are developed as polynomials in a set of four basic generating functions defined on $S_{4}$. These functions were identified as generators of a complete linearly independent basis of $\mathrm{SO}(3)$-coupled wave functions for $L^{2}\left(S_{4}\right)$ [23], based on a knowledge of $L^{2}\left(S_{4}\right)$ as a direct sum of irreducible $\mathrm{SO}(3)$ subspaces [obtained from the $\mathrm{SO}(5) \rightarrow \mathrm{SO}(3)$ branching rules]. The generating functions can be related to the "elementary permissible diagrams" of the well-known algorithm of Chacón et al. $[20,21]$ for construction of a $\mathrm{U}(5) \supset \mathrm{SO}(5) \supset \mathrm{SO}(3)$ basis for the five-dimensional harmonic oscillator, implemented in the nuclear collective model code of Hess et al. $[24,25]$. The salient property of the generating functions for $L^{2}\left(S_{4}\right)[10]$ is that they yield a direct route to the construction of $\mathrm{SO}(5) \supset \mathrm{SO}(3)$ spherical harmonics without reference to the full harmonic oscillator problem. The method is set forth more concretely in Sec. 2.3.

\subsection{Representation of functions on $S_{4}$}

The quadrupole moments $q_{m}(m=0, \pm 1, \pm 2)$ for the collective model transform as components of an $L=2$ spherical tensor under $\mathrm{SO}(3)$ rotations, i.e.,

$$
q_{m} \rightarrow \sum_{k} q_{k} \mathscr{D}_{k m}^{(2)}(\Omega)
$$

where $\Omega$ represents the Euler angles for the $\mathrm{SO}(3)$ rotation, and the Wigner $\mathscr{D}$ function [26] is the rotation matrix element. [The quantities $q_{m}$ can alternatively be taken to represent the nuclear surface deformation parameters $\alpha_{m}(m=0, \pm 1, \pm 2)$. The difference is only in physical interpretation and does not affect the following results for $\mathrm{SO}(5)$.]

These quadrupole moments are conveniently expressed in terms of Bohr's spherical polar coordinates $(\beta, \gamma, \Omega)[27]$, by the relation

$q_{m}=\beta \cos \gamma \mathscr{D}_{0, m}^{(2)}(\Omega)+\frac{1}{\sqrt{2}} \beta \sin \gamma\left[\mathscr{D}_{2, m}^{(2)}(\Omega)+\mathscr{D}_{-2, m}^{(2)}(\Omega)\right]$.

The squared length of a vector $q \in \mathbb{R}^{5}$ is given by $\sum_{m}\left|q_{m}\right|^{2}=\beta^{2}$. Thus, $\beta$ is the radial coordinate for $\mathbb{R}^{5}$, and $(\gamma, \Omega)$ are angular coordinates.

For consideration of the angular functions on the unit sphere $S_{4}$, we henceforth set $\beta=1$ and restrict consideration to the unit length quadrupole moments $\mathcal{Q}_{m}$, defined as

$$
\mathcal{Q}_{m}=\cos \gamma \mathscr{D}_{0, m}^{(2)}(\Omega)+\frac{1}{\sqrt{2}} \sin \gamma\left[\mathscr{D}_{2, m}^{(2)}(\Omega)+\mathscr{D}_{-2, m}^{(2)}(\Omega)\right] .
$$

These unit-length quadrupole moments are then proportional to the basic $v=1$ spherical harmonics on $S_{4}$.

Consider a function $\Psi_{M}^{(L)}(\gamma, \Omega)$ on $S_{4}$, of good $\mathrm{SO}(3)$ angular momentum $L$ and $\mathrm{SO}(2)$ quantum number $M$. Any such function may be expanded [27] in the form

$$
\Psi_{M}^{(L)}(\gamma, \Omega)=\sum_{\substack{K=0 \\ \text { even }}}^{L} F_{K}(\gamma) \xi_{K M}^{(L)}(\Omega)
$$

where

$$
\xi_{K M}^{(L)}(\Omega) \equiv \frac{1}{\left(1+\delta_{K}\right)^{1 / 2}}\left[\mathscr{D}_{K M}^{(L)}(\Omega)+(-)^{L} \mathscr{D}_{-K M}^{(L)}(\Omega)\right] .
$$

Note that the $\mathscr{D}$ functions occur only in the symmetrized linear combinations $\mathscr{D}_{K M}^{(L)}+(-)^{L} \mathscr{D}_{-K M}^{(L)}$ with even values of $K$. Thus, because $\xi_{-K M}^{(L)}=(-)^{L} \xi_{K M}^{(L)}$, we can restrict to $K \geq 0$. Note also that the functions $\xi_{K M}^{(L)}$ vanish identically for $K=0$ when $L$ is odd. The normalization factor $\left(1+\delta_{K}\right)^{-1 / 2}$ (where $\left.\delta_{K} \equiv \delta_{K, 0}\right)$ is included for later convenience.

The functions $\xi_{K M}^{(L)}(\Omega)$, with $K \geq 0$, provide an orthogonal basis for those functions of the $\mathrm{SO}(3)$ angles which respect the symmetry properties of collective model wave functions. From the inner product for the $\mathscr{D}$ functions [26],

$$
\int \mathscr{D}_{K^{\prime} M^{\prime}}^{\left(L^{\prime}\right) *}(\Omega) \mathscr{D}_{K M}^{(L)}(\Omega) d \Omega=\frac{8 \pi^{2}}{2 L+1} \delta_{L^{\prime} L} \delta_{K^{\prime} K} \delta_{M^{\prime} M},
$$

we obtain the inner product for the nonzero $\xi_{K M}^{(L)}(\Omega)$

$$
\int \xi_{K^{\prime} M^{\prime}}^{\left(L^{\prime}\right) *}(\Omega) \xi_{K M}^{(L)}(\Omega) d \Omega=\frac{16 \pi^{2}}{2 L+1} \delta_{L^{\prime} L} \delta_{K^{\prime} K} \delta_{M^{\prime} M} .
$$

The volume element on $S_{4}$ is given by $d v=\sin 3 \gamma d \gamma d \Omega$, where $\gamma$ is integrated over the range $[0, \pi / 3]$. Thus, the inner product $\left\langle\Psi_{2} \mid \Psi_{1}\right\rangle \equiv \int \Psi_{2}^{*} \Psi_{1} \sin 3 \gamma d \gamma d \Omega$ of two functions on $S_{4}$, when expanded according to (7), is given simply by

$$
\left\langle\Psi_{2 M}^{(L)} \mid \Psi_{1 M}^{(L)}\right\rangle=\frac{16 \pi^{2}}{2 L+1} \int\left[\sum_{\substack{K=0 \\ \text { even }}}^{L} F_{1 K}(\gamma) F_{2 K}(\gamma)\right] \sin 3 \gamma d \gamma
$$

Functions of distinct $L$ or $M$ are orthogonal.

\section{3. $\mathrm{SO}(3)$-coupled basis functions}

The generating function method [10] for construction of the $\mathrm{SO}(5) \supset \mathrm{SO}(3)$ spherical harmonics rests upon the 
observation that a complete set of normalizable $\mathrm{SO}(3)$ highest-weight (i.e., those for which $M=L$ ) functions on $S_{4}$ is provided by the products

$$
\begin{aligned}
& \Phi_{\left[n_{1}, n_{2}, n_{3}, n_{4}\right]}(\gamma, \Omega) \\
& =\left[\Phi_{1}(\gamma, \Omega)\right]^{n_{1}}\left[\Phi_{2}(\gamma, \Omega)\right]^{n_{2}}\left[\Phi_{3}(\gamma, \Omega)\right]^{n_{3}}\left[\Phi_{4}(\gamma, \Omega)\right]^{n_{4}},
\end{aligned}
$$

where the exponents $n_{1}, n_{2}$, and $n_{3}$ take on the values 0 , $1, \ldots$, and $n_{4}$ is restricted to 0 or 1 . These $\Phi_{\left[n_{1}, n_{2}, n_{3}, n_{4}\right]}$ are monomials in four generating functions $\Phi_{1}, \Phi_{2}, \Phi_{3}$, and $\Phi_{4}$, which are simply the $M=L$ components

$$
\begin{aligned}
& \Phi_{1} \propto \mathcal{Q}_{2} \\
& \Phi_{2} \propto(\mathcal{Q} \times \mathcal{Q})_{2}^{(2)} \\
& \Phi_{3} \propto(\mathcal{Q} \times \mathcal{Q} \times \mathcal{Q})_{0}^{(0)} \\
& \Phi_{4} \propto(\mathcal{Q} \times \mathcal{Q} \times \mathcal{Q})_{3}^{(3)},
\end{aligned}
$$

of $\mathrm{SO}(3)$-coupled products involving $\mathcal{Q}$. The coupled product of two tensors is defined by

$$
\left[U^{\left(L_{2}\right)} \times T^{\left(L_{1}\right)}\right]_{M}^{(L)} \equiv \sum_{M_{1} M_{2}}\left(\begin{array}{cc|c}
L_{1} & L_{2} & L \\
M_{1} & M_{2} & M
\end{array}\right) U_{M_{2}}^{\left(L_{2}\right)} T_{M_{1}}^{\left(L_{1}\right)}
$$

Note the right-to-left coupling order in this definition of the $\mathrm{SO}(3)$ tensor product, used for consistency with Refs. $[8,10]$ and to simplify the phases [28] arising in the Wigner-Eckart theorem. The norms of these generating functions are arbitrary and can be chosen for convenience. In the standard form (7), we take

$$
\begin{aligned}
& \Phi_{1}(\gamma, \Omega)=\cos \gamma \xi_{02}^{(2)}(\Omega)+\sin \gamma \xi_{22}^{(2)}(\Omega) \\
& \Phi_{2}(\gamma, \Omega)=\cos 2 \gamma \xi_{02}^{(2)}(\Omega)-\sin 2 \gamma \xi_{22}^{(2)}(\Omega) \\
& \Phi_{3}(\gamma, \Omega)=\cos 3 \gamma \xi_{00}^{(0)}(\Omega) \\
& \Phi_{4}(\gamma, \Omega)= \\
& \sin 3 \gamma \xi_{23}^{(3)}(\Omega) .
\end{aligned}
$$

The multiplication of two highest-weight functions yields another highest-weight function (equivalent to the stretched coupling of two angular momentum functions). Hence, every $\Phi_{\left[n_{1}, n_{2}, n_{3}, n_{4}\right]}$ is a highest-weight function, with $L=M=2 n_{1}+2 n_{2}+3 n_{4}$ and "degree" $N=$ $n_{1}+2 n_{2}+3 n_{3}+3 n_{4}$ in the unit quadrupole moments $\mathcal{Q}$ [29]. The multiplication of the basis functions is carried out using the multiplication rule for the highestweight functions $\xi_{K L}^{(L)}$. This rule, which follows from the multiplication rule $[26]$ for $\mathscr{D}$ functions

$$
\begin{aligned}
\mathscr{D}_{K_{2} M_{2}}^{\left(L_{2}\right)}(\Omega) & \mathscr{D}_{K_{1} M_{1}}^{\left(L_{1}\right)}(\Omega) \\
& =\sum_{L}\left(\begin{array}{cc|c}
L_{1} & L_{2} & L \\
K_{1} & K_{2} & K
\end{array}\right)\left(\begin{array}{cc|c}
L_{1} & L_{2} & L \\
M_{1} & M_{2} & M
\end{array}\right) \mathscr{D}_{K M}^{(L)}(\Omega),
\end{aligned}
$$

where $K=K_{1}+K_{2}$ and $M=M_{1}+M_{2}$, is expressed by

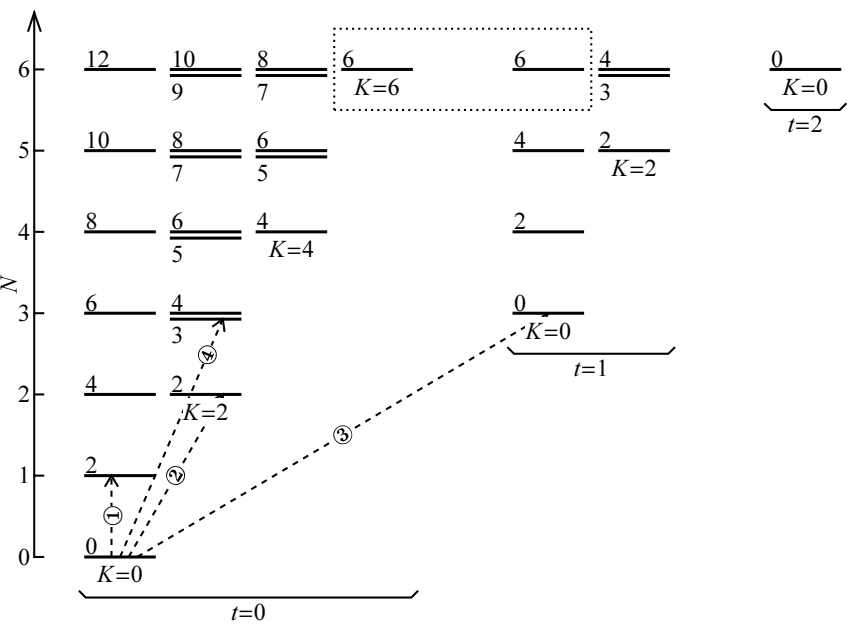

FIG. 1: The set of basis monomials $\Phi_{N t L}$ of degree $N \leq 6$, with labels $N, L, t$, and $K$ indicated. The numbered arrows indicate the raising actions of multiplication by the generating functions $\Phi_{1}, \Phi_{2}, \Phi_{3}$, and $\Phi_{4}$. The angular momentum multiplicity at $N=6$ is highlighted (dotted box). This same diagram enumerates the $\Psi_{v \alpha L}$ with $v \leq 6$, that is, it gives the branching of $\mathrm{SO}(5)$ irreps $(v, 0)$ into angular momenta $L$, if the axis label $N$ is read as $v$.

the equation

$$
\begin{gathered}
\xi_{K_{2} L_{2}}^{\left(L_{2}\right)} \xi_{K_{1} L_{1}}^{\left(L_{1}\right)}=\sum_{K \geq 0} \frac{\left(1+\delta_{K}\right)^{1 / 2}}{\left(1+\delta_{K_{1}}\right)^{1 / 2}\left(1+\delta_{K_{2}}\right)^{1 / 2}} \\
\left.\times\left[\begin{array}{cc|c}
L_{1} & L_{2} & L_{1}+L_{2} \\
K_{1} & K_{2} & K
\end{array}\right)+(-)^{L_{2}}\left(\begin{array}{cc|c}
L_{1} & L_{2} & L_{1}+L_{2} \\
K_{1} & -K_{2} & K
\end{array}\right)\right] \xi_{K, L_{1}+L_{2}}^{L_{1}+L_{2}} .
\end{gathered}
$$

The more general tensor-coupled product of the spherical tensors $\xi_{K}^{(L)}$, which have components $\xi_{K M}^{(L)}$, is considered in Sec. 2.4 .

An equivalent but more descriptive labeling for the highest-weight monomials $\Phi_{\left[n_{1}, n_{2}, n_{3}, n_{4}\right]}(\gamma, \Omega)$ is given by regarding each of them as the $M=L$ component of the set of angular momentum $L$ functions $\Phi_{N t L M}(\gamma, \Omega)$ to which they naturally extend. Here monomials of the same degree $N$ and angular momentum $L$ are distinguished by the label $t \equiv n_{3}$, which counts the number of zero-coupled triplets of the quadrupole coordinates. In addition, the $\Phi$ may be organized into quasibands of given bandhead angular momentum $K=2 n_{2}+2 n_{4}$, as shown in Fig. 1. Together these functions $\Phi_{N t L M}(\gamma, \Omega)$ constitute a spherical tensor $\Phi_{N t L}(\gamma, \Omega)$. Once the highest weight function $\Phi_{N t L L}(\gamma, \Omega)$ has been defined, the remaining $\Phi_{N t L M}(\gamma, \Omega)$ follow immediately, since they share the same coefficients $F_{K}(\gamma)$ in the expansion (7).

An important characteristic of the generating function construction is that the set of $L$ values appearing at a given degree $N$ is identical to the set of $L$ values arising for $\mathrm{SO}(3)$ irreps in an $\mathrm{SO}(5)$ irrep of seniority $v=N$. These are given by the known $\mathrm{SO}(5) \supset \mathrm{SO}(3)$ branching 
TABLE I: The $\mathrm{SO}(5) \supset \mathrm{SO}(3)$ spherical harmonics $\Psi_{v \alpha L}$ for $v \leq 3$. For these lowest-seniority spherical harmonics, orthonormalization is trivial, and the spherical harmonics are simply proportional to the corresponding monomial basis members $\left(\Psi_{v \alpha L}=\mathscr{N}_{v \alpha L}^{-1 / 2} \Phi_{N t L}\right)$. The relations between the $\Psi_{v \alpha L}$ (with $L=0$ and 2 ) and commonly-encountered scalar and quadrupole operators are given in brackets.

\begin{tabular}{|c|c|c|c|}
\hline$v$ & $L$ & $\mathscr{N}_{v \alpha L} /\left(8 \pi^{2}\right)$ & $\left(8 \pi^{2}\right)^{1 / 2} \Psi_{v \alpha L}$ \\
\hline 0 & 0 & $2 / 3$ & $\frac{\sqrt{3}}{2} \xi_{0}^{(0)} \quad\left[=\sqrt{\frac{3}{2}}\right]$ \\
\hline 1 & 2 & $4 / 15$ & $\frac{\sqrt{15}}{2} \cos \gamma \xi_{0}^{(2)}+\frac{\sqrt{15}}{2} \sin \gamma \xi_{2}^{(2)} \quad\left[=\sqrt{\frac{15}{2}} \mathcal{Q}\right]$ \\
\hline \multirow[t]{2}{*}{2} & 4 & $16 / 105$ & $\frac{\sqrt{3}}{8}(7+5 \cos 2 \gamma) \xi_{0}^{(4)}+\frac{3 \sqrt{5}}{4} \sin 2 \gamma \xi_{2}^{(4)}+\frac{\sqrt{105}}{8}(1-\cos 2 \gamma) \xi_{4}^{(4)}$ \\
\hline & 2 & $4 / 15$ & $\frac{\sqrt{15}}{2} \cos 2 \gamma \xi_{0}^{(2)}-\frac{\sqrt{15}}{2} \sin 2 \gamma \xi_{2}^{(2)} \quad\left[=-\frac{\sqrt{105}}{2}(\mathcal{Q} \times \mathcal{Q})^{(2)}\right]$ \\
\hline \multirow[t]{4}{*}{3} & 6 & $32 / 315$ & $\begin{array}{l}\frac{9}{16} \sqrt{\frac{5}{11}}(7 \cos \gamma+\cos 3 \gamma) \xi_{0}^{(6)}+\frac{3}{16} \sqrt{\frac{7}{22}}(15 \sin \gamma+11 \sin 3 \gamma) \xi_{2}^{(6)} \\
\quad+\frac{9}{16} \sqrt{\frac{35}{11}}(\cos \gamma-\cos 3 \gamma) \xi_{4}^{(6)}+\frac{3}{16} \sqrt{\frac{35}{2}}(\sin \gamma-\sin 3 \gamma) \xi_{6}^{(6)}\end{array}$ \\
\hline & 4 & $88 / 945$ & $\frac{3}{4} \sqrt{\frac{3}{22}}(5 \cos \gamma+7 \cos 3 \gamma) \xi_{0}^{(4)}-\frac{9}{2} \sqrt{\frac{5}{22}} \sin \gamma \xi_{2}^{(4)}+\frac{3}{4} \sqrt{\frac{105}{22}}(-\cos \gamma+\cos 3 \gamma) \xi_{4}^{(4)}$ \\
\hline & 3 & $8 / 63$ & $\frac{3}{2} \sqrt{\frac{7}{2}} \sin 3 \gamma \xi_{2}^{(3)}$ \\
\hline & 0 & $4 / 9$ & $\frac{3}{2} \cos 3 \gamma \xi_{0}^{(0)} \quad\left[=\frac{3}{\sqrt{2}} \cos 3 \gamma\right]$ \\
\hline
\end{tabular}

rule $[16,17]$. Thus, Fig. 1 also enumerates the labels of all highest-weight spherical harmonics $\Psi_{v \alpha L L}$ up to the maximum seniority $(v=6)$ shown. The multiplicity $d_{N L}$ of angular momentum $L$ at degree $N$ (also the multiplicity $d_{v L}$ of angular momentum $L$ at seniority $v$ ) is given by (A1). A multiple occurrence of the same $L$ at a given $N$ first arises at $N=6$, for $L=6$ (Fig. 1).

Although the $\Phi_{N t L L}$ form a complete set of highestweight functions and are angular momentum eigenfunctions, they are, in general, non-orthogonal (for a given $L$ ) and also not eigenfunctions of $\hat{\Lambda}^{2}$. Thus, they are not the desired $\mathrm{SO}(5)$ spherical harmonics. Construction of the spherical harmonics $\Psi_{v \alpha L M}(\gamma, \Omega)$ relies on the observation that the highest-weight spherical harmonics $\Psi_{v \alpha L L}$, like the $\Phi_{N t L L}$, form a complete set of highest-weight functions and that they are polynomials of degree $v$ in the unit quadrupole moments $\mathcal{Q}$. For any given $N_{\max }$, the sets $\left\{\Psi_{v \alpha L L} \mid v \leq N_{\max }\right\}$ and $\left\{\Phi_{N t L L} \mid N \leq N_{\max }\right\}$ both span the space of highest-weight polynomials of degree $\leq N_{\max }$ in $\mathcal{Q}$. Construction therefore proceeds inductively, by orthonormalization of the bases of successively higher $N_{\max }$. The $\Psi_{v \alpha L L}$, as polynomials of degree $v$ in $\mathcal{Q}$, are linear combinations of the $\Phi_{N t L L}$ with $N \leq v$. Because they must be orthogonal to all $\Psi_{v^{\prime} \alpha^{\prime} L L}$ of lower seniority $v^{\prime}<v$, they can therefore be obtained by GramSchmidt orthogonalizing the monomials of degree $N=v$ with respect to the space of lower degree.

The orthogonality of the spaces with differing $L$ implies that orthogonalization can be performed separately in each space of given $L$. Within an $L$-space, the spherical harmonics up to seniority $v_{\max }$ are obtained as follows:

(1) Order the basis monomials $\Phi_{N t L L}$, for $0 \leq N \leq$ $v_{\max }$, by increasing $N$, and then by increasing $t$ when multiple basis monomials of the same $L$ occur for a given $N$ [30]. These functions may then be labeled with a single counting index, as $\Phi_{L i}$, with $i=1, \ldots, D_{v_{\max } L}$. The dimension $D_{v_{\max } L}$ of the seniority-truncated $L$ space is given by (A2).

(2) Calculate the overlaps $\left\langle\Phi_{L j} \mid \Phi_{L i}\right\rangle$ for $1 \leq i, j \leq$ $D_{v_{\max } L}$, using (11).

(3) Determine the linear transformation necessary to bring the $\Phi_{L i}$ into an orthonormal set by the GramSchmidt procedure. The result is a matrix of orthogonalization (and normalization) coefficients $T_{L i j}$ for the $L$-space, in terms of which the

$$
\Psi_{L i}=\sum_{j=1}^{D_{v_{\max } L}} T_{L i j} \Phi_{L j}
$$

are the desired spherical harmonics, in order of increasing seniority.

In step (1), if angular momentum multiplicity occurs at a given $N$, the ordering of the $\Phi_{N t L}$ sharing the same $N$ is, in principle, arbitrary. However, choosing a different ordering for the $\Phi_{N t L}$ gives rise, after Gram-Schmidt orthogonalization, to a different, equally valid, set of spherical harmonics at the corresponding seniority $(v=N)$. That is, the spherical harmonics $\Psi_{v \alpha L}\left(\alpha=1, \ldots, d_{v L}\right)$ span a seniority-degenerate subspace, and the ordering of the $\Phi_{N t L}$ in the orthonormalization process determines which of the possible unitarily-equivalent bases for the subspace is selected as the "spherical harmonics".

Note that the overlaps of the $\Phi_{N t L L}$ obey a parity selection rule. Since $\Phi_{N t L L}$ is a product of $N$ factors of 
$\mathcal{Q}$, it has parity $(-)^{N}$ under the $\mathbb{R}^{5}$ parity operation, which takes $\mathcal{Q}_{m} \rightarrow-\mathcal{Q}_{m}$. The overlap of two functions of opposite $\mathbb{R}^{5}$-parity [31] vanishes, so $\left\langle\Phi_{N^{\prime} t^{\prime} L L} \mid \Phi_{N t L L}\right\rangle$ is nonzero only if $N+N^{\prime}$ is even. The $\Psi_{v \alpha L L}$ resulting from the orthonormalization process therefore retain definite $\mathbb{R}^{5}$-parity $(-)^{v}$ and are constructed only from the $\Phi_{N t L L}$ of this same parity.

Hence, by the $\mathbb{R}^{5}$ parity selection rule, all the $\Phi_{N t L M}$ with $N \leq 3$ are already orthogonal and therefore are the spherical harmonics with $v \leq 3$, to within normalization. The necessary normalization factors $\mathscr{N}_{v \alpha L}$, such that $\Psi_{v \alpha L}=\mathscr{N}_{v \alpha L}^{-1 / 2} \Phi_{N t L}$, and resulting spherical harmonics, for $v \leq 3$, are summarized in Table I. The table also provides a glossary relating the $\Psi_{v \alpha L}$ to Hamiltonian $(L=0)$ and electric quadrupole $(L=2)$ operators commonly referenced in physical applications. More generally, even for $v>3$, the two lowest-seniority $\Psi_{v \alpha L}$ for a given value of $L$ are proportional to the $\Phi_{N t L}$ with $N=v$. Other higher-seniority $\Psi_{v \alpha L}$ are nontrivial linear combinations (18) of the $\Phi_{N t L}$ with $N \leq v$.

\subsection{Triple overlap integrals}

For the determination of $\mathrm{SO}(5)$ Clebsch-Gordan coefficients (Sec. 2.5), we need to calculate triple overlap integrals

$$
\left\langle\Psi_{3}\left|\hat{\Psi}_{2}\right| \Psi_{1}\right\rangle \equiv \int \Psi_{3}^{*}(\gamma, \Omega) \Psi_{2}(\gamma, \Omega) \Psi_{1}(\gamma, \Omega) \sin 3 \gamma d \gamma d \Omega .
$$

In this expression, $\hat{\Psi}_{2}$ is interpreted as an operator which acts multiplicatively, i.e., $\hat{\Psi}_{2}\left|\Psi_{1}\right\rangle$ has as its wave function $\Psi_{2}(\gamma, \Omega) \Psi_{1}(\gamma, \Omega)$. For angular momentum coupled functions, we need only the reduced matrix elements $\left\langle\Psi_{3}^{\left(L_{3}\right)}\left\|\hat{\Psi}_{2}^{\left(L_{2}\right)}\right\| \Psi_{1}^{\left(L_{1}\right)}\right\rangle$ defined by the Wigner-Eckart theorem $[26]$

$$
\begin{aligned}
\left\langle\Psi_{3 M_{3}}^{\left(L_{3}\right)}\left|\hat{\Psi}_{2 M_{2}}^{\left(L_{2}\right)}\right| \Psi_{1 M_{1}}^{\left(L_{1}\right)}\right\rangle= & \frac{1}{\left(2 L_{3}+1\right)^{1 / 2}}\left(\begin{array}{cc|c}
L_{1} & L_{2} \\
M_{1} & M_{2} & L_{3} \\
M_{3}
\end{array}\right) \\
& \times\left\langle\Psi_{3}^{\left(L_{3}\right)}\left\|\hat{\Psi}_{2}^{\left(L_{2}\right)}\right\| \Psi_{1}^{\left(L_{1}\right)}\right\rangle
\end{aligned}
$$

where the quantity in parentheses is an $\mathrm{SO}(3)$ ClebschGordan coefficient.

The Wigner-Eckart theorem is easily inverted (e.g., Ref. [8]) by application of the Clebsch-Gordan unitarity condition, to give the reduced matrix element in a computationally convenient form in terms of the coupled action of $\hat{\Psi}_{2}^{\left(L_{2}\right)}$ on $\left|\Psi_{1}^{\left(L_{1}\right)}\right\rangle$. Namely,

$$
\begin{aligned}
& \left\langle\Psi_{3}^{\left(L_{3}\right)}\left\|\hat{\Psi}_{2}^{\left(L_{2}\right)}\right\| \Psi_{1}^{\left(L_{1}\right)}\right\rangle \\
& \quad=\left(2 L_{3}+1\right)^{1 / 2}\left\langle\Psi_{3 M_{3}}^{\left(L_{3}\right)}\right|\left[\hat{\Psi}_{2}^{\left(L_{2}\right)} \times\left|\Psi_{1}^{\left(L_{1}\right)}\right\rangle\right]_{M_{3}}^{\left(L_{3}\right)},
\end{aligned}
$$

where $\left[\hat{\Psi}_{2}^{\left(L_{2}\right)} \times\left|\Psi_{1}^{\left(L_{1}\right)}\right\rangle\right]_{M_{3}}^{\left(L_{3}\right)}$ has wave function

$$
\begin{aligned}
& {\left[\Psi_{2}^{\left(L_{2}\right)}(\gamma, \Omega) \times \Psi_{1}^{\left(L_{1}\right)}(\gamma, \Omega)\right]_{M_{3}}^{\left(L_{3}\right)}=} \\
& \quad \sum_{M_{1} M_{2}}\left(\begin{array}{ll|l}
{ }_{1} L_{1} & L_{2} & L_{2} \\
M_{3}
\end{array}\right) \Psi_{2 M_{2}}^{\left(L_{2}\right)}(\gamma, \Omega) \Psi_{1 M_{1}}^{\left(L_{1}\right)}(\gamma, \Omega),
\end{aligned}
$$

following the convention (14) for the coupled product of tensors. The functions $\xi_{K M}^{(L)}(M=0, \pm 1, \ldots, \pm L)$, regarded as components of a spherical tensor $\xi_{K}^{(L)}$, obey the coupling rule

$$
\begin{aligned}
& {\left[\xi_{K_{2}}^{\left(L_{2}\right)}(\Omega) \times \xi_{K_{1}}^{\left(L_{1}\right)}(\Omega)\right]^{(L)}=\frac{\left(1+\delta_{K_{1}+K_{2}}\right)^{1 / 2}}{\left(1+\delta_{K_{1}}\right)^{1 / 2}\left(1+\delta_{K_{2}}\right)^{1 / 2}}\left(\left.\begin{array}{c}
L_{1} \\
K_{1} K_{2}
\end{array}\right|_{K_{1}+K_{2}} ^{L}\right) \xi_{K_{1}+K_{2}}^{(L)}(\Omega)}
\end{aligned}
$$

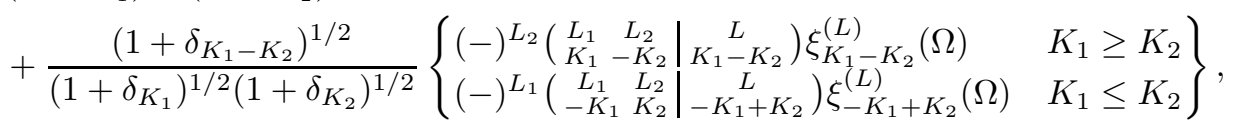

obtained by direct application of (16). It follows that [33]

$$
\begin{aligned}
& \left\langle\Psi_{3}^{\left(L_{3}\right)}\left\|\hat{\Psi}_{2}^{\left(L_{2}\right)}\right\| \Psi_{1}^{\left(L_{1}\right)}\right\rangle=\frac{16 \pi^{2}}{\left(2 L_{3}+1\right)^{1 / 2}} \int\left[\sum_{\substack{K_{1}, K_{2}, K_{3} \\
\text { even }}} \frac{\left(1+\delta_{K_{3}}\right)^{1 / 2}}{\left(1+\delta_{K_{1}}\right)^{1 / 2}\left(1+\delta_{K_{2}}\right)^{1 / 2}}\right.
\end{aligned}
$$

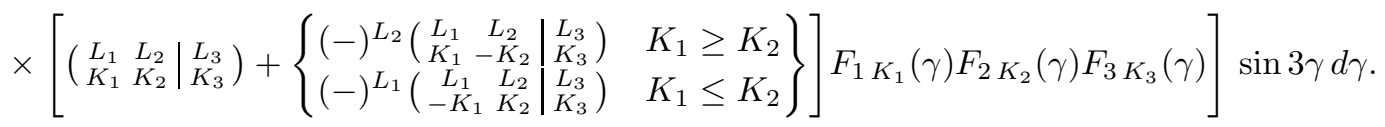

\section{5. $\mathrm{SO}(5) \supset \mathrm{SO}(3)$ Clebsch-Gordan coefficients}

Representations of $\mathrm{SO}(5)$ couple to form new representations according to $\mathrm{SO}(5)$ Clebsch-Gordan coefficients.
If the $\mathrm{SO}(5)$ representations are labeled according to the $\mathrm{SO}(5) \supset \mathrm{SO}(3) \supset \mathrm{SO}(2)$ subalgebra chain (1), each coupling coefficient may be written as the product of an 
$\mathrm{SO}(3)$-reduced Clebsch-Gordan coefficient and an ordinary $\mathrm{SO}(3)$ Clebsch-Gordan coefficient, according to the Racah factorization lemma [34] (see Ref. [35] for a general discussion). We term the $\mathrm{SO}(3)$-reduced factor an $\mathrm{SO}(5) \supset \mathrm{SO}(3)$ Clebsch-Gordan coefficient. Coupling coefficients, such as these, which are reduced with respect to a subalgebra are also known as "isoscalar factors" [36].

In the context of the $\mathrm{SO}(5)$ spherical harmonics, only the symmetric representations $(v, 0)$ of $\mathrm{SO}(5)$ arise.
These are related by the $\mathrm{SO}(5) \supset \mathrm{SO}(3)$ Clebsch-Gordan

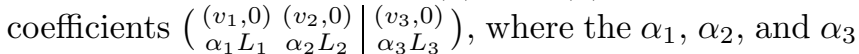
are multiplicity indices, corresponding to the multiplicity in the $\Psi_{v \alpha L}$. Let $\left\{\chi_{\alpha_{1} L_{1} M_{1}}^{\left(v_{1}, 0\right)}\right\}$ and $\left\{\chi_{\alpha_{2} L_{2} M_{2}}^{\left(v_{2}, 0\right)}\right\}$ denote orthonormal bases for $\mathrm{SO}(5)$ representations of seniority $v_{1}$ and $v_{2}$, respectively. Then an orthonormal basis for an $\mathrm{SO}(5)$ tensor-coupled product irrep of seniority $v$ is defined by

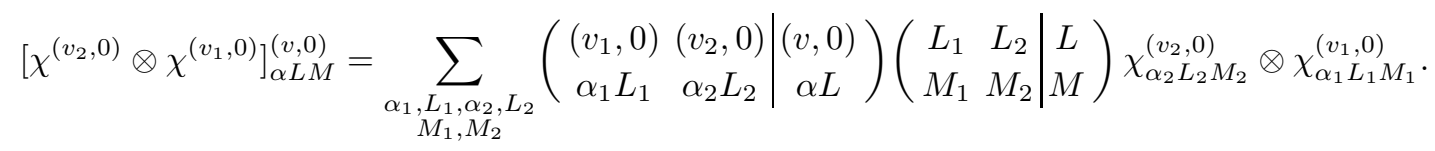

The $\mathrm{SO}(5) \supset \mathrm{SO}(3)$ Clebsch-Gordan coefficients enter into the $\mathrm{SO}(5)$ Wigner-Eckart theorem, which governs matrix elements of $\mathrm{SO}(5) \supset \mathrm{SO}(3)$ tensor operators. The Racah factorization lemma may be used to write the $\mathrm{SO}(5)$ Wigner-Eckart theorem in terms of $\mathrm{SO}(3)$-reduced quantities as

$$
\begin{aligned}
& \left\langle\Psi_{v_{3} \alpha_{3} L_{3}}\left\|\hat{\Psi}_{v_{2} \alpha_{2} L_{2}}\right\| \Psi_{v_{1} \alpha_{1} L_{1}}\right\rangle \\
& =\sqrt{2 L_{3}+1}\left(\begin{array}{cc|c}
\left(v_{1}, 0\right) & \left(v_{2}, 0\right) & \left(v_{3}, 0\right) \\
\alpha_{1} L_{1} & \alpha_{2} L_{2} & \alpha_{3} L_{3}
\end{array}\right)\left\langle\Psi_{v_{3}}\left\|\hat{\Psi}_{v_{2}}\right\| \Psi_{v_{1}}\right\rangle,
\end{aligned}
$$

where $\left\langle\Psi_{v_{3}}\left\|\hat{\Psi}_{v_{2}}\right\| \Psi_{v_{1}}\right\rangle$ is an $\mathrm{SO}(5)$-reduced (doublyreduced) matrix element. The factor $\sqrt{2 L_{3}+1}$ compensates for the corresponding factor absorbed into the definition of the $\mathrm{SO}(3)$-reduced matrix element in (20). The $\mathrm{SO}(5) \supset \mathrm{SO}(3)$ Clebsch-Gordan coefficients satisfy the normalization condition

$$
\sum_{\substack{\alpha_{1} L_{1} \\
\alpha_{2} L_{2}}}\left(\begin{array}{cc|c}
\left(v_{1}, 0\right) & \left(v_{2}, 0\right) \\
\alpha_{1} L_{1} & \alpha_{2} L_{2} & \left(v_{3}, 0\right) \\
\alpha_{3} L_{3}
\end{array}\right)^{2}=1
$$

from unitarity, where the phases of the Clebsch-Gordan coefficients are real by convention. The values for $L$ occurring within a given $\mathrm{SO}(5)$ representation $(v, 0)$, and their multiplicities, are governed by the multiplicity formula (A1). The Clebsch-Gordan coefficients van- ish unless $L_{1}, L_{2}$, and $L_{3}$ satisfy the triangle inequality $\left(\left|L_{1}-L_{2}\right| \leq L_{3} \leq L_{1}+L_{2}\right)$. The values of $v_{1}$, $v_{2}$, and $v_{3}$ likewise must satisfy the triangle inequality $\left(\left|v_{1}-v_{2}\right| \leq v_{3} \leq v_{1}+v_{2}\right)$, as well as the parity constraint that $v_{1}+v_{2}+v_{3}$ be even.

The $\mathrm{SO}(5) \quad \supset \quad \mathrm{SO}(3) \quad$ Clebsch-Gordan coeffi-

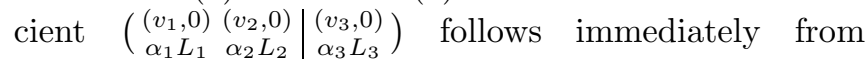
the corresponding $\mathrm{SO}(3)$-reduced matrix element $\left\langle\Psi_{v_{3} \alpha_{3} L_{3}}\left\|\Psi_{v_{2} \alpha_{2} L_{2}}\right\| \Psi_{v_{1} \alpha_{1} L_{1}}\right\rangle$, by (26), once the $\mathrm{SO}(5)$ reduced matrix element $\left\langle\Psi_{v_{3}}\left\|\Psi_{v_{2}}\right\| \Psi_{v_{1}}\right\rangle$ is known. In fact, from (26) and the normalization condition (27), we obtain

$$
\left\langle\Psi_{v_{3}}\left\|\hat{\Psi}_{v_{2}}\right\| \Psi_{v_{1}}\right\rangle^{2}=\sum_{\substack{\alpha_{1} L_{1} \\ \alpha_{2} L_{2}}} \frac{\left\langle\Psi_{v_{3} \alpha_{3} L_{3}}\left\|\hat{\Psi}_{v_{2} \alpha_{2} L_{2}}\right\| \Psi_{v_{1} \alpha_{1} L_{1}}\right\rangle^{2}}{2 L_{3}+1}
$$

for each $L_{3}$ and $\alpha_{3}$. This yields the $\mathrm{SO}(5)$-reduced matrix element in terms of the summed squares of the computed $\mathrm{SO}(3)$-reduced matrix elements, to within an arbitrary phase, which is chosen to be unity.

Explicit closed-form expressions for the $\mathrm{SO}(5)$ reduced matrix element have been given for the case $\left\langle\Psi_{v \pm 1}\left\|\Psi_{1}\right\| \Psi_{v}\right\rangle$, i.e., for the quadrupole tensor [3]. Moreover, by numerical inspection of the normalization sums for an extensive set of computed $\mathrm{SO}(3)$-reduced matrix elements, we find [37] that the $\mathrm{SO}(5)$-reduced matrix elements are given by

$$
\begin{aligned}
\left\langle\Psi_{v_{3}}\left\|\Psi_{v_{2}}\right\| \Psi_{v_{1}}\right\rangle=\frac{1}{4 \pi} \sqrt{\frac{\left(2 v_{1}+3\right)\left(2 v_{2}+3\right)}{\left(v_{3}+2\right)\left(v_{3}+1\right)}} \frac{\left(\frac{1}{2} \sigma+1\right) !}{\left(\frac{1}{2} \sigma-v_{1}\right) !\left(\frac{1}{2} \sigma-v_{2}\right) !\left(\frac{1}{2} \sigma-v_{3}\right) !} \\
\quad \times \sqrt{(\sigma+4) \frac{\left(\sigma-2 v_{1}+1\right) !\left(\sigma-2 v_{2}+1\right) !\left(\sigma-2 v_{3}+1\right) !}{(\sigma+3) !}}
\end{aligned}
$$


with $\sigma \equiv\left(v_{1}+v_{2}+v_{3}\right)$. This conjectured expression has been verified exhaustively for all combinations of values for $v_{1}, v_{2}$, and $v_{3}$ likely to be considered in nuclear collective model calculations. However, we stress that is has not been proved in full generality. Use of the expression (29) for $\left\langle\Psi_{v_{3}}\left\|\Psi_{v_{2}}\right\| \Psi_{v_{1}}\right\rangle$ in the $\mathrm{SO}(5)$ WignerEckart theorem (26) allows each $\mathrm{SO}(5) \supset \mathrm{SO}(3)$ ClebschGordan coefficients to be extracted directly from the corresponding $\mathrm{SO}(3)$-reduced matrix elements (24), without the need to calculate matrix elements for all $L_{1}$ and $L_{2}$ values involved in the normalization condition (27). [The use of (29) in the code is described in Sec. 4.2.] The validity of the conjecture, for given $v_{1}, v_{2}$, and $v_{3}$, may be established by calculating the normalization sum for the underlying computed $\mathrm{SO}(3)$-reduced matrix elements or, equivalently, by explicitly verifying that the extracted coefficients satisfy (27).

The $\mathrm{SO}(5) \supset \mathrm{SO}(3)$ Clebsch-Gordan coefficients obey symmetry relations [10]

$$
\begin{aligned}
& \left(\begin{array}{cc|c}
\left(v_{2}, 0\right) & \left(v_{1}, 0\right) & \left(v_{3}, 0\right) \\
\alpha_{2} L_{2} & \alpha_{1} L_{1} & \alpha_{3} L_{3}
\end{array}\right) \\
& =(-)^{L_{1}+L_{2}-L_{3}}\left(\begin{array}{cc|c}
\left(v_{1}, 0\right) & \left(v_{2}, 0\right) & \left(v_{3}, 0\right) \\
\alpha_{1} L_{1} & \alpha_{2} L_{2} & \alpha_{3} L_{3}
\end{array}\right)
\end{aligned}
$$

and

$$
\begin{aligned}
& \left(\begin{array}{cc|c}
\left(v_{3}, 0\right) & \left(v_{2}, 0\right) & \left(v_{1}, 0\right) \\
\alpha_{3} L_{3} & \alpha_{2} L_{2} & \alpha_{1} L_{1}
\end{array}\right)=(-)^{L_{1}+L_{2}-L_{3}} \\
& \quad \times \sqrt{\frac{d_{v_{1}}\left(2 L_{3}+1\right)}{d_{v_{3}}\left(2 L_{1}+1\right)}}\left(\begin{array}{cc|c}
\left(v_{1}, 0\right) & \left(v_{2}, 0\right) & \left(v_{3}, 0\right) \\
\alpha_{1} L_{1} & \alpha_{2} L_{2} & \alpha_{3} L_{3}
\end{array}\right),
\end{aligned}
$$

where $d_{v}=\frac{1}{6}(v+1)(v+2)(2 v+3)$ is the dimension of the $\mathrm{SO}(5)$ representation $(v, 0)$, obtained from the Weyl formula (e.g., Ref. [35]). Hence, it is only necessary to calculate the Clebsch-Gordan coefficient for one permutation of $\left(v_{1}, v_{2}, v_{3}\right)$.

\section{IMPLEMENTATION}

\subsection{Overview}

The computer code for construction of $\mathrm{SO}(5) \supset \mathrm{SO}(3)$ spherical harmonics and calculation of $\mathrm{SO}(5) \supset \mathrm{SO}(3)$ Clebsch-Gordan coefficients is implemented as a set of packages for Mathematica 6 [15]. Mathematica provides native support for symbolic arithmetic. In the present context, this allows all calculations to be carried out exactly, in terms of expressions involving square roots of rational numbers.

The basic algorithm for the calculation of $\mathrm{SO}(5)>$ $\mathrm{SO}(3)$ Clebsch-Gordan coefficients, as described in Sec. 2, involves three main computational tasks:

(1) construction of the monomials (12) comprising the basis of highest-weight functions,
(2) calculation of the overlaps (11) of these monomials, as needed for the orthonormalization process, and

(3) calculation of triple overlaps (24), as needed for the $\mathrm{SO}(5) \supset \mathrm{SO}(3)$ Clebsch-Gordan coefficients.

For practical implementation, two algorithmic refinements are made to this scheme. These relate to the internal representation of the functions $F_{K}(\gamma)$ (Sec. 3.2) and to the recognition (and full utilization) of extensive redundancies among the calculations involved in evaluating different triple overlap integrals (Sec. 3.3). Together, an efficient treatment of these aspects of the implementation extends the range of applicability of the method (for calculation in exact arithmetic) from a maximal seniority of approximately 10 to seniorities of $\gtrsim 100$, thereby easily yielding more than sufficient $\mathrm{SO}(5) \supset \mathrm{SO}(3)$ ClebschGordan coefficients for nuclear structure calculations.

To understand the considerations underlying the efficient implementation of the algorithm, let us more closely consider the structure of the calculation. The computationally most demanding task is evaluation of the overlap or triple overlap integrals in the $\Phi_{N t L}$ basis. Recall that this involves first constructing the function of $\gamma$ appearing in the integrand of (11) or (24) and then evaluating the integral with respect to $\gamma$. The integrand is built from the generating functions (15), using (12) and (17). The integrand is thus a polynomial in the trigonometric functions $\cos \gamma, \sin \gamma, \cos 2 \gamma, \sin 2 \gamma$, $\cos 3 \gamma$, and $\sin 3 \gamma$ or, therefore, by multiple-angle identities, a polynomial in $\cos \gamma$ and $\sin \gamma$. For the overlap $\left\langle\Phi_{N_{2} t_{2} L M} \mid \Phi_{N_{1} t_{1} L M}\right\rangle$, the resulting polynomial requires powers of $\cos \gamma$ and $\sin \gamma$ as high as $N_{1}+N_{2}$, or, for the triple overlap $\left\langle\Phi_{N_{3} t_{3} L_{3}}\left\|\hat{\Phi}_{N_{2} t_{2} L_{2}}\right\| \Phi_{N_{1} t_{1} L_{1}}\right\rangle$, as high as $N_{1}+N_{2}+N_{3}$. The integral may be evaluated exactly, but simplification of the polynomial and integration with standard symbolic algebra software becomes prohibitively inefficient for the construction of spherical harmonics with $v \gtrsim 10$. Alternatively, attempts at bruteforce numerical integration are hampered by the highlyoscillatory nature of such polynomials in trigonometric functions. Thus, it is seen that evaluation of the overlap integrals is the defining computational challenge.

An effective solution arises from the realization that any polynomial of degree $n$ in $\cos \gamma$ and $\sin \gamma$ can be decomposed as a finite sum of exponentials

$$
f(\gamma)=\sum_{k=-n}^{n} a_{k} e^{i k \gamma}
$$

i.e., as a finite Fourier series, of degree $n$. Integration of exponentials is trivial. Such a Fourier expansion method was, in fact, used in the final version of the code used to calculate the Clebsch-Gordan coefficients tabulated in Ref. [10]. In the present implementation, from the very beginning of the calculation, we simply represent all $F_{K}(\gamma)$ coefficients, starting with those of the integrity basis functions (15), as finite Fourier series (32). That 
is, every function is replaced by a list of Fourier coefficients $\left\{a_{-n}, \ldots, a_{0}, \ldots, a_{n}\right\}$. Then, when the integrand in (11) or (24) is constructed as a function of $\gamma$, it is already manifestly Fourier expanded, and the $\gamma$ integration is straightforward.

\subsection{Fourier series representation of functions}

When the functions of $\gamma$ are represented as Fourier sums (32), there are three basic arithmetic operations which must be carried out on the corresponding sets of Fourier coefficients. Addition of two functions $f(\gamma)$ and $g(\gamma)$ is accomplished by addition of their Fourier coefficients. Multiplication of a function by a constant is simply accomplished by multiplication of the coefficients by that constant. Multiplication of two functions $f(\gamma)$ and $g(\gamma)$ by each other gives rise to a convolution of the coefficients. Specifically, let $f(\gamma)$ and $g(\gamma)$ be given by Fourier sums $f(\gamma)=\sum_{r=-m}^{m} a_{r}\left(e^{i \gamma}\right)^{r}$ and $g(\gamma)=\sum_{s=-n}^{n} b_{s}\left(e^{i \gamma}\right)^{s}$. Expanding the product and collecting like powers of $e^{i \gamma}$ yields the product rule

$$
f(\gamma) g(\gamma)=\sum_{k=-(m+n)}^{m+n} c_{k}\left(e^{i \gamma}\right)^{k}
$$

with the new Fourier coefficients given by

$$
c_{k}=\sum_{t=\max (-2 m-k,-2 n+k)}^{\min (2 m-k, 2 n+k)} a_{\frac{1}{2}(k+t)} b_{\frac{1}{2}(k-t)} .
$$

This is essentially the Fourier convolution theorem, in discrete form.

For real-valued functions which are even in $\gamma$, the coefficients $a_{k}$ are pure real and obey the symmetry condition $a_{-k}=a_{k}$. Similarly, for odd real-valued functions, the coefficients are pure imaginary and obey the symmetry $a_{-k}=-a_{k}$. For instance, the functions arising in the definition of the generating functions (15) are $\cos m \gamma=\left[\left(e^{+i \gamma}\right)^{m}+\left(e^{-i \gamma}\right)^{m}\right] / 2$ and $\sin m \gamma=\left[\left(e^{+i \gamma}\right)^{m}-\right.$ $\left.\left(e^{-i \gamma}\right)^{m}\right] /(2 i)$, which are even and odd, respectively. In either case, only coefficients with $k \geq 0$ need be stored, and only real-valued coefficients are required, provided a factor of $i$ is absorbed into the definition of the series for odd functions.

Thus, we use the representation

$$
f(\gamma)=\left(\frac{1}{i}\right)^{g} \sum_{k=0}^{n} \frac{a_{k}}{1+\delta_{k}}\left[\left(e^{+i \gamma}\right)^{k}+(-)^{g}\left(e^{-i \gamma}\right)^{k}\right]
$$

with $g=0$ for even functions and $g=1$ for odd functions. This is effectively a Fourier cosine series or a Fourier sine series, for the even and odd cases, respectively.

The definite integrals needed for the calculation are of the form $\int_{0}^{\pi / 3} f(\gamma) d \gamma$. Moreover, only odd integrands $(g=1)$ arise in the problem. The definite integral of an odd series (35) on the "sector" $0 \leq \gamma \leq \pi / 3$ is

$$
\int_{0}^{\pi / 3} f(\gamma) d \gamma=\sum_{k=1}^{n} \frac{2 a_{k}}{k}\left[1-\cos \frac{(k \bmod 6) \pi}{3}\right] .
$$

Evaluation of this sum requires only a limited set of trigonometric values, namely, $\cos k \pi / 3(k=0,1, \ldots, 5)$.

The necessary definitions for working with Fourier representations of functions are contained in the subpackage FourierSum. The Fourier sum (35) is represented symbolically by the expression

$$
\text { FourierSum [BohrGamma, } \left.g,\left\{a_{0}, a_{1}, \ldots, a_{n}\right\}\right] \text {, }
$$

where $g$ indicates the symmetry ( $g=0$ or 1$)$, and the $a_{k}$ are the real-valued Fourier coefficients as defined in (35). (The tag BohrGamma simply serves to indicate that the argument is $\gamma$, since the package allows for more general possibilities.) Thus, for example, $\cos 2 \gamma$ is represented as

$$
\text { FourierSum [BohrGamma, } 0,\{0,0,1 / 2\}] \text {. }
$$

The coefficients which arise in the present calculations are rational numbers or square roots of rational numbers, and they are maintained as exact symbolic expressions throughout the calculation.

The package defines addition of two FourierSum expressions, multiplication by a constant, and multiplication of two FourierSum expressions as extensions to the usual Mathematica + and $*$ operations. The function IntegrateSector $[r, f]$ returns the integral given in (36). The function FourierSumToTrig $[f]$ is also provided, to convert FourierSum expressions back into ordinary symbolic expressions in terms of trigonometric functions.

\subsection{Evaluation of matrix elements}

The task of computing matrix elements (triple overlap integrals) of the $\mathrm{SO}(5) \supset \mathrm{SO}(3)$ spherical harmonics is most conveniently carried out by first calculating the matrix elements of the original monomial basis functions $\Phi_{N t L}$, that is, the $\left\langle\Phi_{N_{3} t_{3} L_{3}}\left\|\hat{\Phi}_{N_{2} t_{2} L_{2}}\right\| \Phi_{N_{1} t_{1} L_{1}}\right\rangle$. The desired matrix elements $\left\langle\Psi_{v_{3} \alpha_{3} L_{3}}\left\|\hat{\Psi}_{v_{2} \alpha_{2} L_{2}}\right\| \Psi_{v_{1} \alpha_{1} L_{1}}\right\rangle$ in the orthonormal spherical harmonic basis then follow immediately from the Gram-Schmidt transformation (18). It is simplest to write this using the counting index labeling within an $L$-space, as

$$
\begin{aligned}
& \left\langle\Psi_{L_{3} i_{3}}\left\|\hat{\Psi}_{L_{2} i_{2}}\right\| \Psi_{L_{1} i_{1}}\right\rangle= \\
& \sum_{j_{1} j_{2} j_{3}} T_{L_{3} i_{3} j_{3}} T_{L_{2} i_{2} j_{2}} T_{L_{1} i_{1} j_{1}}\left\langle\Phi_{L_{3} j_{3}}\left\|\hat{\Phi}_{L_{2} j_{2}}\right\| \Phi_{L_{1} j_{1}}\right\rangle .
\end{aligned}
$$

As described in the preceding section, we have transformed the relatively intractable integration problem into the more manageable task of constructing products of 
$F_{K}(\gamma)$ functions in Fourier representation. Therefore, the computational burden now lies primarily in evaluating the discrete Fourier convolutions (33-34). Although the operations involved are in principle simple arithmetic, the number of iterations required for a single convolution is substantial, growing as the square of the degrees of the Fourier sums involved, and the arithmetic itself involves symbolic simplification of expressions involving square roots of rational numbers.

The challenge, therefore, lies in minimizing the number and complexity of the Fourier convolutions which must be evaluated. The problem is best approached by noting that the matrix elements are not to be calculated singly, but rather in aggregate, that is, as the set of all matrix elements of an operator $\hat{\Phi}_{N_{2} t_{2} L_{2}}$ between $L$-spaces $L_{1}$ and $L_{3}$. The integrand in the expression (24) for the matrix element is a sum over products of $F_{K}(\gamma)$ functions, and products of the exact same pairs of $F_{K}(\gamma)$ functions arise, redundantly, in the evaluation of many different matrix elements.

A few straightforward observations allow us to remove these calculational redundancies. Most obviously, every matrix element $\left\langle\Phi_{N_{3} t_{3} L_{3}}\left\|\hat{\Phi}_{N_{2} t_{2} L_{2}}\right\| \Phi_{N_{1} t_{1} L_{1}}\right\rangle$ involving the same ket $\left|\Phi_{N_{1} t_{1} L_{1}}\right\rangle$ and operator $\hat{\Phi}_{N_{2} t_{2} L_{2}}$ will also involve the same products between $F_{K}(\gamma)$ functions from $\Phi_{N_{1} t_{1} L_{1}}(\gamma, \Omega)$ and $\Phi_{N_{2} t_{2} L_{2}}(\gamma, \Omega)$. In the notation of (21), it is therefore advantageous to calculate the coupled action of $\hat{\Phi}_{N_{2} t_{2} L_{2}}$ on any given $\left|\Phi_{N_{1} t_{1} L_{1}}\right\rangle$, namely, $\left[\hat{\Phi}_{N_{2} t_{2} L_{2}} \times\left|\Phi_{N_{1} t_{1} L_{1}}\right\rangle\right]^{\left(L_{3}\right)}$, only once, and to reuse this intermediate result for the matrix element with each bra $\left\langle\Phi_{N_{3} t_{3} L_{3}}\right|$.

More significant, though, is the observation that all powers of $\Phi_{3} \propto \cos 3 \gamma$ factor out of the summations in (21). (This will be more clearly apparent below.) Thus, the integrands involved in the calculation of many different $\left\langle\Phi_{N_{3} t_{3} L_{3}}\left\|\hat{\Phi}_{N_{2} t_{2} L_{2}}\right\| \Phi_{N_{1} t_{1} L_{1}}\right\rangle$, sharing the same total label $t=t_{1}+t_{2}+t_{3}$, are actually identical. Even greater reduction in the number of convolutions needed is obtained by first evaluating the integrand only for those monomials involving no powers of $\Phi_{3}\left(t_{1}=t_{2}=\right.$ $\left.t_{3}=0\right)$ and only then multiplying by the relevant power $(\cos 3 \gamma)^{t}$.

In practice, the entire process is based on operations involving the coefficient functions $F_{K}(\gamma)$ which describe functions on $S_{4}$, as in (7). The relevant definitions are provided by the subpackage WignerDSum. A function on $S_{4}$ is represented as the expression

$$
\text { WignerDSum }\left[L,\left\{F_{0}, F_{2}, \ldots, F_{\lfloor L\rfloor_{2}}\right\}\right],
$$

where $L$ is the angular momentum and $\lfloor L\rfloor_{2}$ denotes the greatest even integer less than or equal to $L$. The $F_{K}$, in turn, are FourierSum expressions (Sec. 3.2). Thus, for example, the generating function $\Phi_{1}$ from (15) is represented by

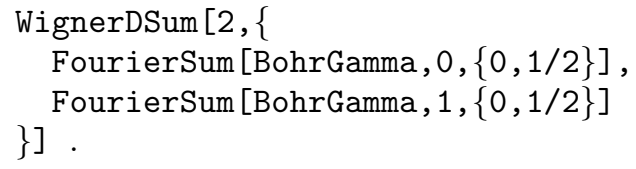

Operations of addition of two functions and multiplication by a constant are readily defined, by entry-wise operations on the lists of $F_{K}(\gamma)$ coefficients. These operations are defined by the package as extensions to the usual Mathematica + and $*$ operations.

To obtain the coupled action $\left[\hat{\Phi}_{N_{2} t_{2} L_{2}} \times\left|\Phi_{N_{1} t_{1} L_{1}}\right\rangle\right]^{\left(L_{3}\right)}$, we evaluate the coupled product $\left[\Phi_{N_{2} t_{2} L_{2}}(\gamma, \Omega) \times\right.$ $\left.\Phi_{N_{1} t_{1} L_{1}}(\gamma, \Omega)\right]^{\left(L_{3}\right)}$. The calculation follows in a straightforward fashion from the coupling rule (23) for the functions $\xi_{K}^{(L)}$. The coupling operation is provided by the WignerDSum package as the function TensorCouple $\left[\Psi_{1}, \Psi_{2}, L\right]$.

However, the monomials $\Phi_{N_{2} t_{2} L_{2}}(\gamma, \Omega)$ and $\Phi_{N_{1} t_{1} L_{1}}(\gamma, \Omega)$ must first themselves be constructed, from the definition (12). As noted in Sec. 2.3, multiplication of highest-weight functions is identical to stretched $\left(L=L_{1}+L_{2}\right)$ tensor coupling. The stretched product rule (17) is simply the $L=L_{1}+L_{2}$ special case of the more general coupling rule (23) for the $\xi_{K}^{(L)}$. Therefore, the $\Phi_{N t L}$ are also constructed in the code by use of this same function TensorCouple, as

$$
\Phi_{N t L}=\left[\left[\Phi_{1}^{(2)}\right]^{n_{1}} \times\left[\Phi_{2}^{(2)}\right]^{n_{2}} \times\left[\Phi_{3}^{(0)}\right]^{n_{3}} \times\left[\Phi_{4}^{(3)}\right]^{n_{4}}\right]^{(L)}
$$

where $\Phi_{1}^{(2)}$ is the tensor with highest-weight component $\Phi_{1}(\gamma, \Omega)$, etc., and $L=2 n_{1}+2 n_{2}+3 n_{4}$ (Sec. 2.3). To complete the calculation of the matrix element, the overlap with $\left\langle\Phi_{N_{3} t_{3} L_{3}}\right|$ must be evaluated. In fact, the function TensorCouple also suffices for this purpose. The sum of products of $F_{K}(\gamma)$ arising in the overlap integral (11) is readily expressed in coupled form. By the coupling rule (23),

$$
\left[\Psi_{2}^{(L)} \times \Psi_{1}^{(L)}\right]_{0}^{(0)}=\frac{2}{(2 L+1)^{1 / 2}} \sum_{\substack{K=0 \\ \text { even }}}^{L} F_{1 K}(\gamma) F_{2 K}(\gamma) .
$$

This follows from the basic expression for $L=$ 0 Clebsch-Gordan coefficients, much like the usual scalar product result $\left[T_{2}^{(L)} \times T_{1}^{(L)}\right]_{0}^{(0)}=(-)^{L}(2 L+$ $1)^{-1 / 2} \sum_{M}(-)^{M} T_{1 M}^{(L)} T_{2-M}^{(L)}$.

It remains then to carry out the integration over $\gamma$, by use of IntegrateSector (Sec. 3.2). If we define $\langle f(\gamma)\rangle \equiv$ $8 \pi^{2} \int_{0}^{\pi / 3} f(\gamma) \sin 3 \gamma d \gamma$, where the factor of $8 \pi^{2}$ results from the integration over Euler angles, then the reduced matrix element deduced from the overlap (21) is simply

$$
\begin{aligned}
& \left\langle\Phi_{N_{3} t_{3} L_{3}}\left\|\Phi_{N_{2} t_{2} L_{2}}\right\| \Phi_{N_{1} t_{1} L_{1}}\right\rangle \\
& \quad=\left\langle\left[\Phi_{N_{3} t_{3} L_{3}} \times\left(\Phi_{N_{2} t_{2} L_{2}} \times \Phi_{N_{1} t_{1} L_{1}}\right)^{\left(L_{3}\right)}\right]_{0}^{(0)}\right\rangle .
\end{aligned}
$$

Expressing the overlap in terms of a coupled product does not change the underlying calculation (and at most affords a convenient economy of coding). However, this formulation does permit the factorization of $(\cos 3 \gamma)^{t}$ from the integrand to be expressed in an especially symmetric (and explicit) form. Observe that $\Phi_{3}^{(0)} \propto \cos 3 \gamma$ is 
a scalar, and, therefore, unlike the other generating functions, it factors out of the coupled product defining the monomial in (38), and subsequently out of the couplings in the zero-coupled product in (40). Hence, if we label each of the monomials by its exponents with respect to each of the generating functions, the integral factorizes as

$$
\begin{gathered}
{\left[\Phi_{\left[n_{1}^{\prime}, n_{2}^{\prime}, n_{3}^{\prime}, n_{4}^{\prime}\right]}^{\left(L_{3}\right)} \times\left(\Phi_{\left[\nu_{1}, \nu_{2}, \nu_{3}, \nu_{4}\right]}^{\left(L_{2}\right)} \times \Phi_{\left[n_{1}, n_{2}, n_{3}, n_{4}\right]}^{\left(L_{1}\right)}\right)^{\left(L_{3}\right)}\right]_{0}^{(0)}} \\
\left.=\left[\Phi_{\left[n_{1}^{\prime}, n_{2}^{\prime}, 0, n_{4}^{\prime}\right]}^{\left(L_{3}\right)} \times\left(\Phi_{\left[\nu_{1}, \nu_{2}, 0, \nu_{4}\right]}^{\left(L_{2}\right)} \times \Phi_{\left[n_{1}, n_{2}, 0, n_{4}\right]}^{\left(L_{1}\right)}\right)\right]_{0}^{\left(L_{3}\right)}\right]_{0}^{(0)} \\
\times \Phi_{[0,0, t, 0]}, \quad(41)
\end{gathered}
$$

where $t=n_{3}^{\prime}+\nu_{3}+n_{3}$ is the total exponent of $\Phi_{3}^{(0)}$ occurring in the product. Note that only monomials of the form $\Phi_{\left[n_{1}, n_{2}, 0, n_{4}\right]}$, with $n_{3}=0$, are actually needed in the calculation, along with $\Phi_{[0,0, t, 0]}=(\sqrt{2} \cos 3 \gamma)^{t}$.

The mechanics of the optimized calculation of matrix elements therefore proceed as follows. The monomials $\Phi_{\left[n_{1}, n_{2}, 0, n_{4}\right]}$ are constructed by (38). To eliminate redundancy, this is done recursively, that is, by multiplication of the monomial of one lower degree in $n_{1}, n_{2}$, or $n_{4}$ by the appropriate generating function, and all the intermediate results are cached. Then the successive coupled products in (41) are constructed and cached: $\left(\Phi_{\left[\nu_{1}, \nu_{2}, 0, \nu_{4}\right]}^{\left(L_{2}\right)} \times \Phi_{\left[n_{1}, n_{2}, 0, n_{4}\right]}^{\left(L_{1}\right)}\right)^{\left(L_{3}\right)}($ stage I $),\left[\Phi_{\left[n_{1}^{\prime}, n_{2}^{\prime}, 0, n_{4}^{\prime}\right]}^{\left(L_{3}\right)} \times\right.$ $\left.\left(\Phi_{\left[\nu_{1}, \nu_{2}, 0, \nu_{4}\right]}^{\left(L_{2}\right)} \times \Phi_{\left[n_{1}, n_{2}, 0, n_{4}\right]}^{\left(L_{1}\right)}\right)^{\left(L_{3}\right)}\right]_{0}^{(0)}$ (stage II), and finally the full integrand with $\Phi_{[0,0, t, 0]}$ included (stage III).

For matrix elements within an $L$-space, Hermiticity reduces the number of independent matrix elements nearly by half. Since, under complex conjugation, $\Phi_{N t L}$ satisfies $\Phi_{N t L M}^{*}=(-)^{L-M} \Phi_{N t L-M}$, it follows [26] that

$$
\begin{aligned}
& \left\langle\Phi_{N_{3} t_{3} L_{3}}\left\|\hat{\Phi}_{N_{2} t_{2} L_{2}}\right\| \Phi_{N_{1} t_{1} L_{1}}\right\rangle \\
& \quad=(-)^{L_{3}+L_{2}-L_{1}}\left\langle\Phi_{N_{1} t_{1} L_{1}}\left\|\hat{\Phi}_{N_{2} t_{2} L_{2}}\right\| \Phi_{N_{3} t_{3} L_{3}}\right\rangle^{*} .
\end{aligned}
$$

As a specific example of the computational savings provided by the caching of intermediate products, consider the calculation of matrix elements of $\Phi_{112} \propto \mathcal{Q}$ within the space $L_{1}=L_{3}=40$, for all monomials of degree $\leq 50$. [This calculation yields the $\mathrm{SO}(5) \supset \mathrm{SO}(3)$ ClebschGordan coefficients with $v_{2}=1$ and $L_{2}=2$, for all $v_{1}$ and $v_{3} \leq 50$.] There are $D_{50,40}=154$ basis functions and thus 23716 matrix elements to be calculated, or 11935 after the relation (42) is taken into account. Evaluation of the coupled action of $\Phi_{112}$ (stage I) involves only 21 couplings, since the coupling is only carried out for basis functions with $n_{3}=0$. Evaluation of the scalar couplings (stage II) constitutes the bulk of the calculation. Whereas each of the couplings evaluated in stage I involves a low- $L$, low-degree factor $\left(\Phi_{112}\right)$, in stage II both factors, $\Phi_{\left[n_{1}^{\prime}, n_{2}^{\prime}, 0, n_{4}^{\prime}\right]}^{\left(L_{3}\right)}$ and $\left(\Phi_{\left[\nu_{1}, \nu_{2}, 0, \nu_{4}\right]}^{\left(L_{2}\right)} \times \Phi_{\left[n_{1}, n_{2}, 0, n_{4}\right]}^{\left(L_{1}\right)}\right)^{\left(L_{3}\right)}$, are typically of high $L$ (hence each contains many $F_{K}$ terms) and high degree with respect to $\gamma$ (hence many terms are involved in the Fourier sums). A total of 231

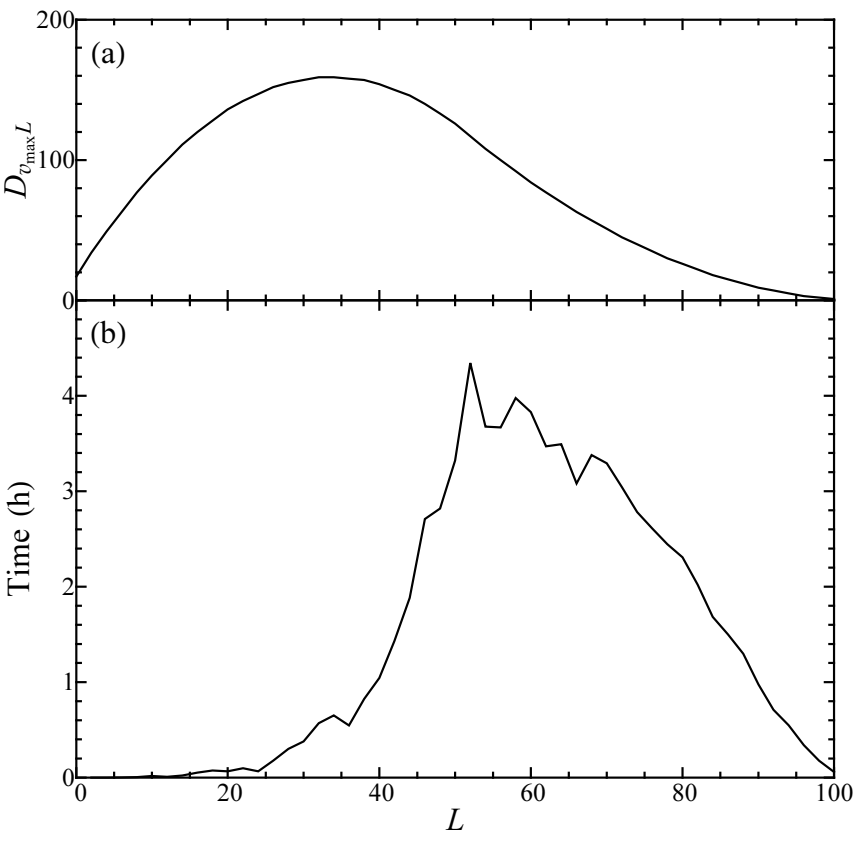

FIG. 2: Basis sizes and corresponding execution times, for calculating the matrix elements of $\Psi_{112} \propto \mathcal{Q}$ between the spherical harmonics with $v \leq 50$. (a) The number of basis functions $D_{v_{\max } L}\left(v_{\max }=50\right)$, for $L$ even. (b) Time for evaluating the matrix elements within a single $L$-space $\left(L_{1}=L_{3} \equiv L\right)$, again only shown for $L$ even. (The basis dimensions and, consequently, execution times are significantly smaller for $L$ odd.) All times are for calculations carried out under Mathematica 6 for Linux, running on a $2.2 \mathrm{GHz}$ Advanced Micro Devices Opteron processor.

such couplings are necessary. Many more distinct expressions, namely, 3001, must be evaluated at stage III, but each entails only a single multiplication of scalar functions (Fourier convolution) and is therefore not computationally intensive.

Total execution times for evaluation of these matrix elements within each $L$-space, as well as the dimensions of these spaces, are shown in Fig. 2. It is seen that calculations for higher $L$ are essentially more demanding than those for lower $L$, even if the bases for the $L$-spaces are of the same size. For higher $L$, a larger number of $F_{K}$ terms are involved in each calculation. Furthermore, the basis monomials tend to involve higher powers of those generating functions which carry angular momentum $\left(\Phi_{1}^{(2)}\right.$, $\Phi_{2}^{(2)}$, and $\left.\Phi_{4}^{(3)}\right)$, in preference to $\Phi_{3}^{(0)}$, lessening the extent to which the optimizations discussed above can simplify the calculation.

The full, optimized process of evaluating the matrix elements of a monomial $\Phi_{N_{2} t_{2} L_{2}}$ between two $L$-spaces $L_{1}$ and $L_{3}$ is carried out by the function CalculateMonomialMatrix. The considerations just described also apply to eliminating redundancies in the evaluation of the overlaps needed for the orthonormalization process. 


\section{USE OF THE COMPUTER CODE}

\subsection{Installation}

The Computer Physics Communications Program Library deposit associated with this article contains Mathematica package files GammaHarmonic.m, FourierSum.m, WignerDSum.m, GramSchmidt.m, and Cache.m, in ASCII text format. Annotated code is included for the packages in both Mathematica notebook format and Portable Document Format. The deposit also includes a Mathematica notebook demonstrating the use of the code (Example.nb) and example output files containing tabulations of Clebsch-Gordan coefficients (see Sec. 4.2).

All package files must be placed in a directory in the Mathematica file search path, as explained in the Mathematica documentation [15], or else in the current working directory for Mathematica. The package GammaHarmonic must then be loaded, by evaluating Get["GammaHarmonic"], as demonstrated in Example.nb.

\subsection{Instructions}

The GammaHarmonic package provides calculation control functions to carry out the several steps involved in constructing and tabulating a set of $\mathrm{SO}(5) \supset \mathrm{SO}(3)$ Clebsch-Gordan coefficients. The syntax for each of the control functions is given in Table II. Here, we provide basic instructions for carrying out these calculational tasks. A worked example, with sample input and output, is given in the Mathematica notebook Example.nb.

(1) The labeling scheme for the basis monomials must first be set up, using ConstructQNSet (see Table II for the appropriate syntax). This function assembles and stores a list of $(N, L, t, K)$ labels for the monomials $(N \leq$ $\left.v_{\max }\right)$ spanning each $L$-space $\left(L \leq L_{\max }\right)$ [38].

(2) The necessary monomials $\Phi_{N t L}$ for the calculation must be constructed, with ConstructMonomials. Only monomials with $n_{3}=0$ are evaluated, for the reasons discussed in Sec. 3.3.

(3) The function ConstructMonomialMatrices is then used to construct matrix elements $\left\langle\Phi_{L_{3} i_{3}}\left\|\hat{\Phi}_{L_{2} i_{2}}\right\| \Phi_{L_{1} i_{1}}\right\rangle$ in the monomial basis, by the process discussed in Sec. 3.3. The function calculates all matrix elements for a given $L_{2}$ and $i_{2}$, between all $L$-spaces $L_{1}$ and $L_{3}$, up to $L_{\max }$, subject to the triangle inequality. This calculation should be carried out for any $\left(L_{2}, i_{2}\right)$ which contribute, in (37), to the final spherical harmonic matrix elements of interest. For nuclear structure applications, these would typically include $\left(L_{2}, i_{2}\right)=(2,1)$, for the electric quadrupole operator, and $\left(L_{2}, i_{2}\right)=(0,2)$, needed for $\cos ^{n} 3 \gamma$ contributions to the potential in the Hamiltonian. The calculation must also be carried out for $\left(L_{2}, i_{2}\right)=(0,1)$, i.e., for the identity operator $\Phi_{010}$, since this provides the overlaps needed for the Gram-Schmidt orthonormalization.

(4) The function ConstructBasisGST must be used to generate the Gram-Schmidt transformation coefficients $T_{L i j}$.

(5) Finally, the function ConstructBasisMatrices is used to calculate the reduced matrix elements $\left\langle\Psi_{L_{3} i_{3}}\left\|\hat{\Psi}_{L_{2} i_{2}}\right\| \Psi_{L_{1} i_{1}}\right\rangle$ of the spherical harmonics, by Gram-Schmidt transformation (37) of the previouslycalculated monomial matrix elements.

The $\mathrm{SO}(5) \supset \mathrm{SO}(3)$ Clebsch-Gordan coefficients follow from these computed $\mathrm{SO}(3)$-reduced matrix elements, by the $\mathrm{SO}(5)$ Wigner-Eckart theorem (26) and the normalization condition (27), as described in Sec. 2.5. Individual Clebsch-Gordan coefficients may be accessed with the function S05ClebschGordan [ $v_{\max }$, $\left.\left\{v_{1}, L_{1}, \alpha_{1}\right\},\left\{v_{2}, L_{2}, \alpha_{2}\right\},\left\{v_{3}, L_{3}, \alpha_{3}\right\}\right]$. So that the coefficients can be extracted independently, without requiring computation of matrix elements for all $L_{1}$ and $L_{2}$ values encountered in the normalization sum (27), the function S05ClebschGordan directly applies the expression (29) for the $\mathrm{SO}(5)$-reduced matrix element in the $\mathrm{SO}(5)$ Wigner-Eckart theorem.

The function WriteS05CGTable outputs a tabulation of $\mathrm{SO}(5) \supset \mathrm{SO}(3)$ Clebsch-Gordan coefficients sharing the same $v_{2}, \alpha_{2}$, and $L_{2}$. These correspond to spherical harmonic matrix elements sharing the same $\hat{\Psi}_{v_{2} \alpha_{2} L_{2}}$. Coefficients are listed in order of increasing $L_{3}, L_{1}, v_{3}, \alpha_{3}$, $v_{1}$, and $\alpha_{1}$ (that is, with the last of these indices varying most rapidly). Only coefficients with $L_{1} \leq L_{3}$ are tabulated, in recognition of the symmetry relation (31), and only those coefficients for which nonzero values are allowed under the $\mathrm{SO}(3)$ triangle inequality and $\mathrm{SO}(5)$ selection rules (Sec. 2.5) are included.

The Clebsch-Gordan coefficients are written both exactly and as floating point numbers. The exact value of a Clebsch-Gordan coefficient can be expressed as the signed square root of a rational number, $\pm \sqrt{a / b}$. The magnitude of each value is squared in the output, to eliminate the need for radicals, so the value written is $\pm a / b$. Because the numerators and denominators appearing in these rational numbers can be large (exceeding 400 decimal digits, for instance, for $v_{\max }=50$ ), the floating point value is also given. This is meant to facilitate input by external programs written in languages which do not provide native support for arbitrary-length integers. Each row of the tabulation has the form

$$
\begin{array}{lllllllllll}
v_{1} & L_{1} & \alpha_{1} & v_{2} & L_{2} & \alpha_{2} & v_{3} & L_{3} & \alpha_{3} & x & \pm a / b,
\end{array}
$$

where $x$ is the floating point representation of $\pm a / b$.

Tabulated coefficients may be read back with ReadS05CGTable. The corresponding SO(3)-reduced matrix elements are recovered and stored as matrices BasisOperatorMatrix $\left[v_{\max },\left\{L_{2}, i_{2}\right\},\left\{L_{3}, L_{1}\right\}\right]$, which may then be used, for instance, for ACM calculations in Mathematica. When using the matrix elements calculated by the code, it should be noted that the factor of $8 \pi^{2}$ which arises from the integration over Euler angles has been suppressed in the actual calculations. (This choice is 
TABLE II: Control functions for $\mathrm{SO}(5) \supset \mathrm{SO}(3)$ spherical harmonic and $\mathrm{SO}(5) \supset \mathrm{SO}(3)$ Clebsch-Gordan coefficient generation.

\begin{tabular}{|c|c|}
\hline Function & Description \\
\hline ConstructQNSet $\left[v_{\max }, L_{\max }\right]$ & $\begin{array}{l}\text { Constructs a list of monomial }(N, L, t, K) \text { labels for each } \\
L \text {-space. }\end{array}$ \\
\hline ConstructMonomials $\left[v_{\max }, L_{\max }\right]$ & Precaches the $\Phi_{N t L}$ constituting the monomial basis. \\
\hline ConstructMonomialMatrices $\left[v_{\max }, L_{\max },\left\{L_{2}, i_{2}\right\}\right]$ & $\begin{array}{l}\text { Calculates the matrix elements }\left\langle\Phi_{L_{3} i_{3}}\left\|\hat{\Phi}_{L_{2} i_{2}}\right\| \Phi_{L_{1} i_{1}}\right\rangle \text { in the } \\
\text { monomial basis, by evaluation of the triple overlap integrals. }\end{array}$ \\
\hline ConstructBasisGST $\left[v_{\max }, L_{\max }\right]$ & Constructs the Gram-Schmidt transformation coefficients. \\
\hline ConstructBasisMatrices $\left[v_{\max }, L_{\max },\left\{L_{2}, i_{2}\right\}\right]$ & $\begin{array}{l}\text { Calculates the matrix elements }\left\langle\Psi_{L_{3} i_{3}}\left\|\Psi_{L_{2} i_{2}}\right\| \Psi_{L_{1} i_{1}}\right\rangle \text { in the } \\
\text { spherical harmonic basis, by Gram-Schmidt transformation of } \\
\text { the monomial matrix elements. }\end{array}$ \\
\hline WriteS05CGTable $\left[v_{\max }, L_{\max },\left\{L_{2}, i_{2}\right\}\right.$, filename $]$ & $\begin{array}{l}\text { Writes a tabulation of } \mathrm{SO}(5) \supset \mathrm{SO}(3) \text { Clebsch-Gordan } \\
\text { coefficients, as defined in the text, including both the exact } \\
\text { rational and floating-point values. }\end{array}$ \\
\hline ReadS05CGTable $\left[v_{\max }, L_{\max },\left\{L_{2}, i_{2}\right\}\right.$, filename $]$ & $\begin{array}{l}\text { Reads back a tabulation of } \mathrm{SO}(5) \supset \mathrm{SO}(3) \text { Clebsch-Gordan } \\
\text { coefficients, as defined in the text. }\end{array}$ \\
\hline
\end{tabular}

made in order to eliminate the symbol $\pi$ from input and output.) If the true normalization is desired, all computed matrix elements must be multiplied by $\left(8 \pi^{2}\right)^{-1 / 2}$.

Two example tabulations of $\mathrm{SO}(5) \supset \mathrm{SO}(3)$ ClebschGordan coefficients are included with the Computer Physics Communications Program Library deposit. These contain coefficients with $\left(v_{2}, L_{2}\right)=(1,2)$ and $(3,0)$ [that is, $\left(L_{2}, i_{2}\right)=(2,1)$ and $\left.(0,2)\right]$, for all seniorities $v_{1}$ and $v_{3}$ up to $v_{\max }=50$ and for all allowed values of $L_{1}$ and $L_{3}$ at these seniorities $\left(L_{\max }=100\right)$. The tabulations are given in the files basis-50-100-cg-2-1. dat and basis-50-100-cg-0-2.dat. (The numerical labels indicate $v_{\max }, L_{\max }, L_{2}$, and $i_{2}$, respectively.) The tabulated coefficients are of sufficient extent to support basic nuclear structure calculations with the ACM.

Intermediate results may be saved to files at various stages of the calculation, and later retrieved, allowing computations to be resumed or extended [39] at a later time. In particular, monomial matrix elements may be saved with WriteMonomialMatrices and subsequently read back with ReadMonomialMatrices (see Example.nb and the internal program documentation). Similar functions are defined to write and read back the GramSchmidt transformation coefficients and to write out the $\mathrm{SO}(3)$-reduced matrix elements of the spherical harmonics in matrix format.

\subsection{Explicit expressions for the $\mathrm{SO}(5) \supset \mathrm{SO}(3)$ spherical harmonics}

Explicit expressions for the $\mathrm{SO}(5) \supset \mathrm{SO}(3)$ spherical harmonics, as functions of $\gamma$ and the Euler angles (Table I), are not needed for diagonalization of the collective model Hamiltonian or calculation of transition matrix elements in the ACM framework. The $\mathrm{SO}(5) \supset \mathrm{SO}(3)$ Clebsch-Gordan coefficients suffice. However, if ACM calculations are carried out in an $\mathrm{SU}(1,1) \times \mathrm{SO}(5)$ basis, probability distributions $P(\beta, \gamma)$ for the wave functions in coordinate space can then be obtained by combining the known radial wave functions $R_{n}^{\lambda}(\beta)$ [3] and the expressions for the $\Psi_{v \alpha L}(\gamma, \Omega)$. The general procedure is given in the appendix of Ref. [9], and example probability distributions may be found in Figs. 3 and 4 of that reference.

The function ConstructBasisWaveFunctions explicitly constructs the spherical harmonics $\Psi_{v \alpha L}(\gamma, \Omega)$, by taking linear combinations of the monomials $\Phi_{N t L}$, according to the Gram-Schmidt transformation (18). The spherical harmonics may be converted from Fourier representation to symbolic expressions involving trigonometric functions by use of FourierSumToTrig (Sec. 3.2), as demonstrated in Example.nb. A normalization factor of $\left(8 \pi^{2}\right)^{-1 / 2}$ must be restored in these results, as indicated in Table I, if the true normalization is desired.

\subsection{Algebraic collective model infrastructure}

If ACM calculations are to be carried out within Mathematica, the GammaHarmonic package provides several additional functions which can facilitate this process. As already noted (Sec. 4.2), the package provides the necessary function (ReadS05CGTable) for input of previously-calculated $\mathrm{SO}(5) \supset \mathrm{SO}(3)$ Clebsch-Gordan coefficients. The function TruncateBasisMatrices may then be used to truncate the stored matrices of $\mathrm{SO}(3)$ reduced matrix elements to lower $v_{\max }$, in order to reduce the product space dimensions for ACM calculations. The GammaHarmonic package also defines several functions (S05LSpaceSize, S05Branching, S05LSpaceSeniorities, etc.) based on the $\mathrm{SO}(5) \supset \mathrm{SO}(3)$ dimension and branching formulas of Appendix A (see the internal program documentation). These functions are of use in indexing the $\mathrm{SU}(1,1) \times \mathrm{SO}(5)$ basis functions and constructing the seniority contribution to the kinetic energy operator. 


\section{CONCLUSION}

The computational methods described in this article, as implemented in the accompanying computer code, make possible the calculation of a sufficient set of $\mathrm{SO}(5) \supset$ $\mathrm{SO}(3)$ Clebsch-Gordan coefficients to support fully converged nuclear structure calculations with the algebraic collective model $(\mathrm{ACM})$. The $\mathrm{SU}(1,1) \times \mathrm{SO}(5)$ algebraic structure of the ACM basis permits matrix elements of an essentially unlimited set of potential and kinetic energy operators to easily be constructed. The Bohr collective model in the resulting calculational scheme is thus genuinely an algebraic collective model. The availability of $\mathrm{SO}(5) \supset \mathrm{SO}(3)$ Clebsch-Gordan coefficients, in conjunction with a large body of analytic expressions for $\beta$ matrix elements $[3,4]$, makes it possible to algebraically construct the matrix elements for any collective model Hamiltonian expressible as a polynomial in the collective quadrupole moments and canonical momenta. Algebraic expressions for matrix elements of a variety of other operators, including the term $\beta^{-2}$ occurring in the Davidson potential $[40,41]$, have also been derived [3, 4].

The calculated $\mathrm{SO}(5) \supset \mathrm{SO}(3)$ Clebsch-Gordan coefficients now permit the diagonalization of the Bohr Hamiltonian for potentials of essentially arbitrary $\gamma$ stiffness. This allows application of the ACM to the full range of nuclear quadrupole rotational-vibrational structure, from spherical oscillator to axial rotor to triaxial rotor. With an appropriate optimized choice of $\beta$ basis functions [3], fully converged calculations can be carried out very efficiently, providing a valuable tool for studying collective motion in nuclei.

The $\mathrm{SU}(1,1) \times \mathrm{SO}(5)$ framework also opens the door for more detailed exploration of the formal relationship between the Bohr collective model and the interacting boson model (IBM) [11], through the $\mathrm{U}(6) \supset \mathrm{U}(5) \supset \mathrm{SO}(5)$ and $\mathrm{U}(6) \supset \mathrm{SO}(6) \supset \mathrm{SO}(5)$ bases. Algebraic collective model methods have already been applied [13] to calculation of collective states within the $\mathrm{SO}(6)$ limit of the
IBM. The availability $\mathrm{SO}(5) \supset \mathrm{SO}(3)$ Clebsch-Gordan coefficients enables such studies to be enhanced and extended.

\section{Acknowledgments}

We thank J. Repka for helpful discussion. This work was supported by the US DOE under grant DE-FG0295ER-40934 and by the Natural Sciences and Engineering Research Council of Canada.

\section{APPENDIX A: BRANCHING AND MULTIPLICITY FOR THE REDUCTION $\mathrm{SO}(5) \supset \mathrm{SO}(3)$}

The multiplicity of each $\mathrm{SO}(3)$ irrep $(L)$ within the $\mathrm{SO}(5)$ irrep $(v, 0)$ is needed in the code, both to determine the $\mathrm{SO}(5) \supset \mathrm{SO}(3)$ branching for the basis and to convert between the $(v \alpha L)$ and $(L i)$ labeling schemes. The result of Refs. [7, 42] can be expressed more compactly as

$$
d_{v L}=\left(\left\lfloor\frac{1}{3}(v-b)\right\rfloor+1\right) \theta_{v-b}-\left\lfloor\frac{1}{3}(v-L+2)\right\rfloor \theta_{v-L+2},
$$

where $b \equiv L / 2$ for $L$ even or $(L+3) / 2$ for $L$ odd. The step function $\theta_{k}$ is unity for $k \geq 0$ and zero otherwise. The dimension $D_{v_{\max } L} \equiv \sum_{v=0}^{v_{\max }} d_{v L}$ of the basis for a given $L$-space, truncated at seniority $v \leq v_{\max }$, is consequently

$$
\begin{array}{r}
D_{v_{\max } L}=\left[f\left(v_{\max }-b, 3\right)+\left(v_{\max }-b+1\right)\right] \theta_{v_{\max }-b} \\
-f\left(v_{\max }-L+2,3\right) \theta_{v_{\max }-L+2},
\end{array}
$$

where $f(n, m) \equiv \sum_{k=0}^{n}\lfloor k / m\rfloor$ is given by

$$
f(n, m)=\lfloor n / m\rfloor\left[n+1-\frac{1}{2} m(\lfloor n / m\rfloor+1)\right] .
$$

[1] A. Bohr and B. R. Mottelson, Nuclear Structure, Vol. 2 (World Scientific, Singapore, 1998).

[2] D. J. Rowe, Nucl. Phys. A 735 (2004) 372.

[3] D. J. Rowe and P. S. Turner, Nucl. Phys. A 753 (2005) 94.

[4] D. J. Rowe, J. Phys. A 38 (2005) 10181.

[5] G. Gneuss, U. Mosel, and W. Greiner, Phys. Lett. B 30 (1969) 397.

[6] G. Gneuss, U. Mosel, and W. Greiner, Phys. Lett. B 31 (1970) 269.

[7] J. M. Eisenberg and W. Greiner, Nuclear Theory, 3rd ed., Vol. 1 (North-Holland, Amsterdam, 1987).

[8] D. J. Rowe and J. L. Wood, Fundamentals of Nuclear Models: Foundational Models (World Scientific, Singapore, in press).

[9] M. A. Caprio, Phys. Rev. C 72 (2005) 054323.

[10] D. J. Rowe, P. S. Turner, and J. Repka, J. Math. Phys.
45 (2004) 2761.

[11] F. Iachello and A. Arima, The Interacting Boson Model (Cambridge University Press, Cambridge, 1987).

[12] F. Iachello and P. Van Isacker, The Interacting BosonFermion Model (Cambridge University Press, Cambridge, 1991).

[13] D. J. Rowe and G. Thiamova, Nucl. Phys. A 760 (2005) 59.

[14] M. A. Caprio and F. Iachello, Nucl. Phys. A 781 (2007) 26.

[15] Wolfram Research, Inc., Mathematica 6 (Champaign, Illinois, 2007).

[16] N. Kemmer, D. L. Pursey, and S. A. Williams, J. Math. Phys. 9 (1968) 1224.

[17] S. A. Williams and D. L. Pursey, J. Math. Phys. 9 (1968) 1230.

[18] L. Wilets and M. Jean, Phys. Rev. 102 (1956) 788. 
[19] D. R. Bès, Nucl. Phys. 10 (1959) 373.

[20] E. Chacón, M. Moshinsky, and R. T. Sharp, J. Math. Phys. 17 (1976) 668.

[21] E. Chacón and M. Moshinsky, J. Math. Phys. 18 (1977) 870

[22] S. De Baerdemacker, K. Heyde, and V. Hellemans, J. Phys. A 40 (2007) 2733.

[23] The generating functions that we use can also be derived by methods similar to those used in deriving an "integrity basis" [43] for the $\mathrm{SO}(3)$ invariants in the universal enveloping algebra of $\mathrm{SU}(3)$ and by construction of a generating function for the character of the reducible $\mathrm{SO}(3)$ representation on $L^{2}\left(S_{4}\right)$ [44].

[24] P. O. Hess, M. Seiwert, J. Maruhn, and W. Greiner, Z. Phys. A 296 (1980) 147.

[25] D. Troltenier, P. O. Hess, and J. A. Maruhn, in Computational Nuclear Physics 1: Nuclear Structure, edited by K. Langanke, J. A. Maruhn, and S. E. Koonin (SpringerVerlag, Berlin, 1991), p. 105.

[26] A. R. Edmonds, Angular Momentum in Quantum Mechanics, 2nd ed., Investigations in Physics No. 4 (Princeton University Press, Princeton, New Jersey, 1960).

[27] A. Bohr, Mat. Fys. Medd. Dan. Vid. Selsk. 26 (14)(1952).

[28] Use of left-to-right coupling (e.g., Ref. [26]) for the $\mathrm{SO}(3)$ tensor product would introduce phase factors of the form $(-)^{L_{1}+L_{2}-L_{3}}$ in some of the intermediate results (namely, those which explicitly involve the tensor coupling operation) of the following sections. However, the choice of convention has no effect on the final computed matrix elements or $\mathrm{SO}(5) \supset \mathrm{SO}(3)$ Clebsch-Gordan coefficients.

[29] Strictly speaking, the degree of an arbitrary polynomial in the components $\mathcal{Q}_{m}$ of the quadrupole tensor is not well-defined. Since $\mathcal{Q} \cdot \mathcal{Q} \equiv \sum_{m}\left|\mathcal{Q}_{m}\right|^{2}=1$, a polynomial may be multiplied by arbitrary factors of $\mathcal{Q} \cdot \mathcal{Q}$ with no effect. The definition of degree must therefore be restricted to polynomials that cannot be reduced to polynomials of lower degree by means of the identity $\mathcal{Q} \cdot \mathcal{Q}=1$. This condition is automatically satisfied by the highest-weight functions obtained as products (12) of generating functions.

[30] See, however, endnote [38] regarding the ordering of monomials of the same $L$ and $N$.

[31] The $\mathbb{R}^{5}$-parity quantum number has variously been termed $\gamma$-parity [19], $d$-parity [32], and $q$-parity [10] in different nuclear collective model contexts.

[32] N. Pietralla, P. von Brentano, A. Gelberg, T. Otsuka, A. Richter, N. Smirnova, and I. Wiedenhöver, Phys. Rev. C 58 (1998) 191.

[33] The expression (24) is equivalent to equation (A4) of Ref. [9]. However, in comparing the expressions, note that Ref. [9] follows a different normalization convention for the reduced matrix element in the Wigner-Eckart theorem and for the coefficients $F_{K}(\gamma)$.

[34] G. Racah, Phys. Rev. 76 (1949) 1352.

[35] B. G. Wybourne, Classical Groups for Physicists (Wiley, New York, 1974).

[36] A. R. Edmonds, Proc. R. Soc. London A 268 (1962) 567.

[37] D. J. Rowe, T. A. Welsh, and M. A. Caprio (in preparation).

[38] The order in which the labels are stored determines the order of orthogonalization in the Gram-Schmidt procedure. By default, the convention of Sec. 2.3 is followed in the case of multiplicities, namely, monomials are orthonormalized in order of increasing $t$. However, the opposite ordering (decreasing $t$ ) was used for the tabulation in Ref. [10], which yields a unitarily equivalent but distinct set of values. The code will employ the latter convention if the option Order->-1 is given to ConstructQNSet.

[39] If calculations have already been completed through $L_{\max }=L_{\max , 0}$, they may be continued from this point onward (to higher $L_{\max }$ ) by specifying the option Last $->L_{\max , 0}$ to any of the calculation control functions (Table II) or input/output functions. Alternatively, by this mechanism, calculations for different sets of $L$-spaces may be carried out concurrently on multiple processors or systems.

[40] J. P. Elliott, J. A. Evans, and P. Park, Phys. Lett. B 169 (1986) 309.

[41] D. J. Rowe and C. Bahri, J. Phys. A 31 (1998) 4947.

[42] T. Kishimoto and T. Tamura, Nucl. Phys. A 163 (1971) 100.

[43] B. R. Judd, W. Miller, Jr., J. Patera, and P. Winternitz, J. Math. Phys. 15 (1974) 1787.

[44] T. A. Welsh and D. J. Rowe (in preparation). 